\title{
Skolemization and Herbrand Theorems for Lattice-Valued Logics
}

\author{
Petr Cintula ${ }^{1}$ \\ Institute of Computer Science, Czech Academy of Sciences \\ Prague, Czech Republic \\ Denisa Diaconescu \\ Faculty of Mathematics and Computer Science, University of Bucharest \\ Bucharest, Romania \\ George Metcalfe ${ }^{2}$ \\ Mathematical Institute, University of Bern \\ Bern, Switzerland
}

\begin{abstract}
Skolemization and Herbrand theorems are obtained for first-order logics based on algebras with a complete lattice reduct and operations that are monotone or antitone in each argument. These lattice-valued logics, defined as consequence relations on inequations between formulas, typically lack properties underlying automated reasoning in classical first-order logic such as prenexation, deduction theorems, or reductions from consequence to satisfiability. Skolemization and Herbrand theorems for the logics therefore take various forms, applying to the left or right of consequences, and restricted classes of inequations. In particular, in the presence of certain witnessing conditions, they admit sound "parallel" Skolemization procedures where a strong quantifier is removed by introducing a finite disjunction or conjunction of formulas with new function symbols. A general expansion lemma is also established that reduces consequence in a lattice-valued logic between inequations containing only strong occurrences of quantifiers on the left and weak occurrences on the right to consequence between inequations in the corresponding propositional logic. If propositional consequence is finitary, this lemma yields a Herbrand theorem for the logic.
\end{abstract}

Keywords: Skolemization, Herbrand Theorems, Non-Classical Logics, Lattices

\section{Introduction}

In this paper, we investigate key properties for automated reasoning in first-order lattice-valued logics. The latticevalued approach introduced here provides a general framework for studying nonclassical first-order logics defined via algebras with a complete lattice reduct and operations that are monotone or antitone in each argument, covering not only broad families of first-order many-valued logics, intermediate logics, and substructural logics (studied in, e.g., [10, 13, 15, 18, 23, 25]), but also cases such as lightweight description logics (viewed as fragments of first-order logics) that lack an implication connective suitable for expressing the order (see, e.g., [1]). Logical consequence is defined between inequations consisting of pairs of first-order formulas, encompassing also, via a simple translation, consequence between equations, and, if the language contains a suitable implication connective, consequence between formulas 3

\footnotetext{
Email addresses: cintula@cs.cas.cz (Petr Cintula), ddiaconescu@fmi.unibuc.ro (Denisa Diaconescu), george.metcalfe@math.unibe.ch (George Metcalfe)

${ }^{1}$ Supported by the Czech Science Foundation grant GBP202/12/G061 and RVO 67985807.

${ }^{2}$ Supported by the Swiss National Science Foundation grant 200021_165850 and the EU Horizon 2020 research and innovation programme under the Marie Skłodowska-Curie grant agreement No 689176.

${ }^{3}$ Note that we use the term "lattice-valued logic" here in a much broader sense than [26] and related works, which treat a very particular class of logics.
} 
In classical first-order logic, questions of validity and consequence reduce to the satisfiability of a set of sentences in prenex form; Skolemization and Herbrand theorems then reduce these questions further to the satisfiability of a set of propositional formulas (see, e.g., [5]). More precisely, a prenex sentence $(\forall \bar{x})(\exists y) \varphi(\bar{x}, y)$ is classically satisfiable if and only if $(\forall \bar{x}) \varphi(\bar{x}, f(\bar{x}))$ is satisfiable, where $f$ is a function symbol not occurring in $\varphi$. The satisfiability of a sentence in prenex form hence reduces to the satisfiability of a universal sentence; Herbrand's theorem then yields a further reduction to the satisfiability of a (typically infinite) set of propositional formulas by substituting universally quantified variables with closed terms. For non-classical logics, the situation is not so straightforward. First, due to the absence of certain quantifier shifts, formulas may not be equivalent to prenex formulas, and, second, consequence may not reduce to satisfiability. Hence we consider inequations between non-prenex sentences as premises and conclusions of consequences. Skolemization and Herbrand theorems then take various forms, applying to either the left or right of the consequence relation, and in some cases only to restricted sets of formulas.

Skolemization procedures that remove strong occurrences of quantifiers in subformulas on the left of consequences, and weak occurrences on the right, are not sound in general. For example, $(\forall x) \neg \neg P(x) \rightarrow \neg \neg(\forall x) P(x)$ is not valid in first-order intuitionistic logic, but its Skolem form $(\forall x) \neg \neg P(x) \rightarrow \neg \neg P(c)$ is valid (see, e.g., [2]). However, in some cases, soundness can be regained using an alternative (in the sense of [21]) "parallel Skolemization" procedure. The key idea is to remove strong occurrences of quantifiers on the left of the consequence relation and weak occurrences of quantifiers on the right by introducing disjunctions and conjunctions, respectively, of formulas with multiple new function symbols. In particular, a sentence $(\forall \bar{x})(\exists y) \varphi(\bar{x}, y)$ may be rewritten for some $n \in \mathbb{N}^{+}$ as $(\forall \bar{x}) \bigvee_{i=1}^{n} \varphi\left(\bar{x}, f_{i}(\bar{x})\right)$ where each function symbol $f_{i}$ is new for $i=1, \ldots, n$. This method has been used in [3] to establish Skolemization results for first-order intermediate logics whose Kripke models admit a finite model property, and in [8] to obtain similar results for families of substructural and many-valued logics.

In this paper, we provide general parallel Skolemization theorems for first-order lattice-valued logics that cover and extend these previous results. We show in particular that a lattice-valued logic has parallel Skolemization of degree $n \in \mathbb{N}^{+}$(that is, allowing at most $n$ new function symbols for each step and applying to formulas on both sides of the consequence relation), whenever it admits the " $n$-witnessed model property", a generalization of a property introduced by Hájek in [19] stating, informally, that consequence in the logic is equivalent to consequence in models where quantified subformulas are witnessed by meets or joins of at most $n$ elements. Moreover, we show that when consequence in the lattice-valued logic is finitary, then the converse also holds. Since the $n$-witnessed model property is a rather strong condition, we also consider lattice-valued logics that satisfy a weaker $n$-prewitnessed model property and admit parallel Skolemization on the right of the consequence relation of degree $n$ for inequations between prenex sentences.

Finally, we obtain Herbrand theorems for reducing consequences between inequations without strong occurrences of quantifiers to consequences between propositional inequations, generalizing similar results for substructural logics obtained in [9]. The basis for these theorems is a general expansion lemma that performs this reduction on the left of consequences at a cost of introducing infinitely many propositional inequations. We then prove that finitarity with respect to propositional consequence corresponds exactly to the removal of weak occurrences of quantifiers using only finitely many propositional inequations. Our main Herbrand theorem then establishes, analogously to the classical case, that this reduction can be performed by taking finite conjunctions or disjunctions of instances of formulas. Since consequence cannot be reduced in general to satisfiability in lattice-valued logics, we provide also a Herbrand theorem for satisfiability.

\section{Lattice-Valued Logics}

In this section we introduce the semantics of first-order lattice-valued logics based on classes of algebras whose operations are monotonic increasing or decreasing in each argument according to the polarities of a given signature. These polarities are also used to identify strong and weak occurrences of subformulas and to define the notions of g-universal and g-existential formulas and inequations, generalizing the usual notions of universal and existential formulas. We establish the expected monotonicity properties for the replacement of strong or weak occurrences of subformulas, and prove that satisfaction properties for g-universal and g-existential formulas are preserved under taking substructures of a model. These results allow us to establish that consequences between g-universal inequations on the left and a g-existential inequation on the right can be reduced to consequences with quantifier-free inequations on both sides. 


\subsection{Syntax and Semantics}

A signature with polarities $\mathcal{L}$ (henceforth, simply signature) consists of a countable set $C_{\mathcal{L}}$ of symbols (called connectives), where each connective $c$ has an assigned arity $n_{c} \in \mathbb{N}$ and polarity $p_{c}:\left\{1,2, \ldots, n_{c}\right\} \rightarrow\{-,+\}$. It is called lattice-oriented if $C_{\mathcal{L}}$ contains two binary connectives $\vee$ and $\wedge$ with $p_{\vee}(i)=p_{\wedge}(i)=+$ for $\left.i \in\{1,2\}\right|^{4}$

Given any lattice-oriented signature $\mathcal{L}$, an $\mathcal{L}$-lattice (also known as a lattice with monotone operations, see, e.g., [14, 16]) is an algebraic structure $\boldsymbol{A}=\left\langle A,\left\{c^{\mathcal{A}}\right\}_{c \in C_{\mathcal{L}}}\right\rangle$ satisfying

(i) $\left\langle A, \vee^{A}, \wedge^{A}\right\rangle$ is a lattice with an order defined by $x \leq^{A} y \Leftrightarrow x \wedge^{A} y=x$;

(ii) $c^{A}$ is an $n_{c}$-ary operator on $A$ for each $c \in C_{\mathcal{L}}$ such that for $1 \leq i \leq n_{c}$,

- if $p_{c}(i)=+$, then $c^{A}$ is monotone in the $i$-th argument

- if $p_{c}(i)=-$, then $c^{A}$ is antitone in the $i$-th argument.

We call $\boldsymbol{A}$ complete if $\bigvee^{A} X$ and $\wedge^{A} X$ exist in $A$ for all $X \subseteq A$, and a chain if $x \leq^{A} y$ or $y \leq^{A} x$ for all $x, y \in A$. As usual, we omit superscripts when these are clear from the context.

Example 1. A suitable lattice-oriented signature $\mathcal{L}_{1}$ for a broad family of substructural logics consists of binary connectives $\vee, \wedge, \&$, and $\rightarrow$, and nullary connectives $\overline{0}$ and $\overline{1}$, where the non-trivial polarity functions are defined by

$$
p_{c}(1)=\left\{\begin{array}{ll}
+ & \text { if } c \in\{\vee, \wedge, \&\} \\
- & \text { if } c=\rightarrow
\end{array} \text { and } \quad p_{c}(2)=+ \text { for } c \in\{\vee, \wedge, \&, \rightarrow\}\right.
$$

Suppose now that $\boldsymbol{A}=\langle A, \vee, \wedge, \&, \rightarrow, \overline{0}, \overline{1}\rangle$ is an $\mathcal{L}_{1}$-lattice. If $\langle A, \&, \overline{1}\rangle$ is a commutative monoid and $\rightarrow$ is the residuum of \& (i.e., $x \& y \leq z \Leftrightarrow x \leq y \rightarrow z$ for all $x, y, z \in A$ ), then $\boldsymbol{A}$ is an $\mathrm{FL}_{\mathrm{e}}$-algebra, also known as a commutative pointed residuated lattice.

The class of $\mathrm{FL}_{\mathrm{e}}$-algebras forms a variety that serves as an algebraic semantics for the Full Lambek Calculus with exchange (see, e.g., [15]); algebraic semantics for other substructural logics are then subvarieties of $\mathrm{FL}_{\mathrm{e}}$-algebras. In particular, a $\mathrm{FL}_{\mathrm{e}}$-algebra $\boldsymbol{A}$ where $\overline{0}$ and $\overline{1}$ are the least and greatest elements of the lattice, respectively, is

- a Heyting algebra if $\&=\wedge$;

- an MTL-algebra if $(x \rightarrow y) \vee(y \rightarrow x)=\overline{1}$ for all $x, y \in A$;

- $a$ BL-algebra if it is an MTL-algebra and $x \wedge y=x \&(x \rightarrow y)$ for all $x, y \in A$;

- a Gödel algebra if it is both a BL-algebra and a Heyting algebra;

- an MV-algebra if it is a BL-algebra and $x=(x \rightarrow \overline{0}) \rightarrow \overline{0}$ for all $x, y \in A$.

The varieties of Heyting, MTL, BL, Gödel, and MV-algebras yield algebraic semantics for intuitionistic logic, monoidal t-norm logic, basic fuzzy logic, Gödel logic, and Łukasiewicz logic, respectively. Let us note here also that the Gödel algebra whose lattice reduct is the real unit interval $[0,1]$ with the usual order is called the standard Gödel algebra and denoted by $[0,1]_{\mathrm{G}}$; analogously, an MV-algebra with the same reduct and operation \& defined as $\max (0, x+y-1)$ is called the standard MV-algebra and denoted by $[0,1]_{\mathrm{t}}$.

A (countable) predicate language $\mathcal{P}$ is a triple $\langle\mathbf{P}, \mathbf{F}, \mathbf{a r}\rangle$ consisting of disjoint countable sets $\mathbf{P}$ and $\mathbf{F}$ of predicate and function symbols, respectively, and a function ar: $\mathbf{P} \cup \mathbf{F} \rightarrow \mathbb{N}$ assigning arities to these symbols. We call nullary function symbols object constants and nullary predicate symbols propositional atoms. For convenience, a predicate language containing only propositional atoms will be called propositional.

Let us fix now a lattice-oriented signature $\mathcal{L}$ and a predicate language $\mathcal{P}=\langle\mathbf{P}, \mathbf{F}, \mathbf{a r}\rangle$. We define $\mathcal{P}$-terms, atomic $\mathcal{P}_{\text {-formulas, }}$ and $\langle\mathcal{L}, \mathcal{P}\rangle$-formulas as in classical logic using a fixed countably infinite set $O V$ of object variables $x, y, \ldots$, the quantifiers $\forall$ and $\exists$, and the connectives in $\mathcal{L}$.

\footnotetext{
${ }^{4}$ The notion of a signature with polarities appears in work of Dunn and co-authors in the context of gaggle theory (see, e.g., [13), in the study of order-algebraizability initiated by Pigozzi [24], and in duality theory [16 17]. Howeover, the vast majority of this work concerns only propositional logics and is not directly relevant to the results reported in this paper.
} 
An $\langle\mathcal{L}, \mathcal{P}\rangle$-inequation is a pair of $\langle\mathcal{L}, \mathcal{P}\rangle$-formulas written $\varphi \leq \psi$. For two $\langle\mathcal{L}, \mathcal{P}\rangle$-formulas $\varphi$ and $\psi$, we define $\varphi \approx \psi:=\{\varphi \leq \psi, \psi \leq \varphi\}$. A set of $\langle\mathcal{L}, \mathcal{P}\rangle$-inequations is called an $\langle\mathcal{L}, \mathcal{P}\rangle$-theory.

The notions of bound and free variables, closed terms, sentences, prenex formulas, and substitutability in formulas and inequations are defined in the standard way. In particular, an inequation $\varphi \leq \psi$ is quantifier-free, prenex, or sentential if the formulas $\varphi$ and $\psi$ are, respectively, quantifier-free, prenex, or sentences.

We omit the symbols for signatures or predicate languages (and analogously, other notions parameterized by such symbols) when these are clear from the context. We typically denote inequations by capital Greek letters, e.g., $\Phi, \Psi, \Upsilon, \ldots$, and theories by Latin letters $T, S, \ldots$, often dropping external brackets when a theory contains just one inequation.

Instead of $\chi_{1}, \ldots, \chi_{n}$ (where the $\chi_{i}$ are terms, formulas, or inequations, and $n \in \mathbb{N}$ is arbitrary or fixed by the context) we sometimes write just $\vec{\chi}$. Unless stated otherwise, by the notation $\varphi(\vec{z})$ (respectively, $\Phi(\vec{z}))$ we signify that all free variables of $\varphi$ (respectively, of both formulas in an inequation $\Phi$ ) are among those in the vector of pairwise different object variables $\vec{z}$. If $\chi\left(x_{1}, \ldots, x_{n}, \vec{z}\right)$ is a formula or an inequation and we replace all free occurrences of $x_{i}^{\prime} \mathrm{s}$ in $\chi$ by terms $t_{i}$, we denote the result in the context simply by $\chi\left(t_{1}, \ldots, t_{n}, \vec{z}\right)$.

An $\langle\mathcal{L}, \mathcal{P}\rangle$-structure $\subseteq$ is a pair $\langle\boldsymbol{A}, \mathbf{S}\rangle$ such that

(i) $\boldsymbol{A}$ is a complete $\mathcal{L}$-lattice;

(ii) $\mathbf{S}$ is a triple $\left\langle S,\left\{P^{\mathbf{S}}\right\}_{P \in \mathbf{P}},\left\{f^{\mathbf{S}}\right\}_{f \in \mathbf{F}}\right\rangle$ where

$-S$ is a non-empty set;

$-P^{\mathbf{S}}: S^{n} \rightarrow A$ is a function for each $n$-ary predicate symbol $P \in \mathbf{P}$

$-f^{\mathbf{S}}: S^{n} \rightarrow S$ is a function for each $n$-ary function symbol $f \in \mathbf{F}$.

An $\mathfrak{S}$-evaluation is a mapping $\mathrm{v}: O V \rightarrow S$. For any $\mathfrak{S}$-evaluation $\mathrm{v}$, we denote by $\mathrm{v}[x \rightarrow a]$ the $\mathfrak{\subseteq}$-evaluation satisfying $\mathrm{v}[x \rightarrow a](x)=a$ and $\mathrm{v}[x \rightarrow a](y)=\mathrm{v}(y)$ for each $y \neq x$. Terms and formulas are evaluated in $\subseteq$ with respect

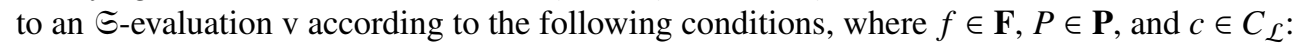

$$
\begin{aligned}
& \|x\|_{\mathrm{v}}^{\mathfrak{E}}=\mathrm{v}(x), \\
& \left\|f\left(t_{1}, \ldots, t_{n}\right)\right\|_{\mathrm{v}}^{\widetilde{E}}=f^{\mathbf{S}}\left(\left\|t_{1}\right\|_{\mathrm{v}}^{\mathbb{E}}, \ldots,\left\|t_{n}\right\|_{\mathrm{v}}^{\mathbb{E}}\right), \\
& \left\|P\left(t_{1}, \ldots, t_{n}\right)\right\|_{\mathrm{v}}^{\mathfrak{E}}=P^{\mathbf{S}}\left(\left\|t_{1}\right\|_{\mathrm{v}}^{\mathfrak{E}}, \ldots,\left\|t_{n}\right\|_{\mathrm{v}}^{\mathbb{E}}\right), \\
& \left\|c\left(\varphi_{1}, \ldots, \varphi_{n}\right)\right\|_{\mathrm{v}}^{\mathfrak{E}}=c^{A}\left(\left\|\varphi_{1}\right\|_{\mathrm{v}}^{\mathbb{E}}, \ldots,\left\|\varphi_{n}\right\|_{\mathrm{v}}^{\mathfrak{⿷}}\right), \\
& \|(\forall x) \varphi\|_{\mathrm{v}}^{\Xi}=\bigwedge\left\{\|\varphi\|_{\mathrm{v}[x \rightarrow a]}^{\Xi} \mid a \in S\right\}, \\
& \|(\exists x) \varphi\|_{\mathrm{v}}^{\subseteq}=\bigvee\left\{\|\varphi\|_{\mathrm{v}[x \rightarrow a]}^{\subseteq} \mid a \in S\right\} .
\end{aligned}
$$

To simplify notation, for a formula $\varphi\left(x_{1}, \ldots, x_{n}\right)$ and an $\Xi$-evaluation $\mathrm{v}$ with $\mathrm{v}\left(x_{i}\right)=a_{i}$ for $1 \leq i \leq n$, we write $\left\|\varphi\left(a_{1}, \ldots, a_{n}\right)\right\|^{\Xi}$ instead of $\left\|\varphi\left(x_{1}, \ldots, x_{n}\right)\right\|_{\mathrm{v}}^{\Xi}$.

For a theory $T$, we say that a structure $\subseteq$ is a model of $T$ and write $\subseteq \vDash T$ if for all $\varphi \leq \psi \in T$ and every S-evaluation v,

$$
\|\varphi\|_{\mathrm{v}}^{\Xi} \leq^{A}\|\psi\|_{\mathrm{v}}^{\Xi} .
$$

Note in particular that $\subseteq \vDash \varphi \approx \psi$ if and only if $\|\varphi\|_{\mathrm{v}}^{\subseteq}=\|\psi\|_{\mathrm{v}}^{\subseteq}$ for every $\subseteq$-evaluation $\mathrm{v}$.

Let us fix now an arbitrary class $\mathbb{K}$ of complete $\mathcal{L}$-lattices and an $\langle\mathcal{L}, \mathcal{P}\rangle$-theory $T \cup\{\Phi\}$. We say that

- an $\langle\mathcal{L}, \mathcal{P}\rangle$-structure $\mathfrak{M}=\langle\boldsymbol{A}, \mathbf{M}\rangle$ is a $\mathbb{K}$-model of $T$ if $\boldsymbol{A} \in \mathbb{K}$ and $\mathfrak{M} \vDash T$;

- an inequation $\Phi$ is a consequence of $T$ with respect to $\mathbb{K}$, written $T \models_{\mathbb{K}} \Phi$, if for each $\mathbb{K}$-model $\mathfrak{M}$ of $T$, also $\mathfrak{M} \vDash \Phi$.

For simplicity, we shorten ' $\{\boldsymbol{A}\}$-model' to ' $\boldsymbol{A}$-model'. Notice also that, since each theory comes with a fixed predicate language, we do not need to specify the language of $\mathfrak{M}$ when we say that it is a model of a theory $T$. For two $\langle\mathcal{L}, \mathcal{P}\rangle$-theories $T$ and $S$, we write $T \models_{\mathbb{K}} S$ if $T \models_{\mathbb{K}} \Phi$ for each $\Phi \in S$.

We list the following (easily confirmed) valid inequations and consequences. 
Lemma 1. Let $\varphi, \psi, \chi$ be $\langle\mathcal{L}, \mathcal{P}\rangle$-formulas.

(a) $\models_{\mathbb{K}} \chi \leq \chi$.

(b) $\{\varphi \leq \psi, \psi \leq \chi\} \models_{\mathbb{K}} \varphi \leq \chi$.

(c) $\models_{\mathbb{K}} \psi(t, \vec{y}) \leq(\exists x) \psi(x, \vec{y})$ if t is substitutable for $x$ in $\psi$.

(d) $\models_{\mathbb{K}}(\forall x) \psi(x, \vec{y}) \leq \psi(t, \vec{y})$ if $t$ is substitutable for $x$ in $\psi$.

(e) $\{\chi \leq \psi\} \models_{\mathbb{K}} \chi \leq(\forall x) \psi$ if $x$ is not free in $\chi$.

(f) $\{\psi \leq \chi\} \models_{\mathbb{K}}(\exists x) \psi \leq \chi$ if $x$ is not free in $\chi$.

Remark 1. In the literature on non-classical logics, it is more common to define consequence on formulas. Consider, e.g., a class $\mathbb{K}$ of complete $\mathrm{FL}_{\mathrm{e}}$-algebras (see Example 1 . According to the usual definition, a structure $\subseteq=\langle\boldsymbol{A}, \mathbf{S}\rangle$ is

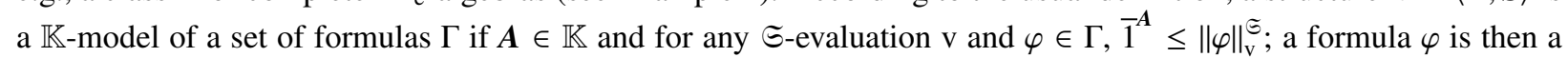
consequence of $\Gamma$ with respect to $\mathbb{K}$, written $\Gamma \models_{\mathbb{K}} \varphi$, if each $\mathbb{K}$-model of $\Gamma$ is a $\mathbb{K}$-model of $\{\varphi\}$. It is easy to see that we can translate between consequences on formulas and inequations as follows:

$$
\begin{aligned}
\Gamma \models_{\mathbb{K}} \varphi & \Longleftrightarrow\{\overline{1} \leq \chi \mid \chi \in \Gamma\} \models_{\mathbb{K}} \overline{1} \leq \varphi \\
T \models_{\mathbb{K}} \varphi \leq \psi & \Longleftrightarrow\{\chi \rightarrow \delta \mid \chi \leq \delta \in T\} \models_{\mathbb{K}} \varphi \rightarrow \psi .
\end{aligned}
$$

Indeed, these translations work whenever there is a binary connective $\rightarrow$ and constant $\overline{1}$ in our lattice-oriented signature $\mathcal{L}$ such that

(a) the notion of a $\mathbb{K}$-model of a set of formulas is defined using $\overline{1}^{A}$ as above;

(b) for each $\mathcal{L}$-lattice $\boldsymbol{A}$ in $\mathbb{K}$, we have $x \leq y \Leftrightarrow \overline{1}^{A} \leq x \rightarrow^{A} y$.

Let us conclude this section by recalling one further important notion. We say that $\models_{\mathbb{K}}$ is finitary if for any $\langle\mathcal{L}, \mathcal{P}\rangle$-theory $T \cup\{\Psi\}$,

$$
T \vDash_{\mathbb{K}} \Psi \Longleftrightarrow T^{\prime} \vDash_{\mathbb{K}} \Psi \text { for some finite } T^{\prime} \subseteq T .
$$

It is easy (using the compactness theorem of first-order logic) to see that if $\mathbb{K}$ is a finite class of finite algebras $\mathbb{K}$, then $\models_{\mathbb{K}}$ is finitary. Below, we formulate further sufficient conditions for the finitarity of $\mathbb{K}$ which follows from known results about axiomatizations of consequence between formulas and the translation mentioned in the previous remark; for details, see, e.g., [11], where an example is also provided demonstrating that these conditions are not necessary.

Proposition 1. Let $\mathcal{L}$ be a signature extending $\mathcal{L}_{1}$ and let $\mathbb{K}$ be a class of $\mathcal{L}$-algebras whose $\mathcal{L}_{1}$-reducts are $\mathrm{FL}_{\mathrm{e}}-$ algebras. Then $\models_{\mathbb{K}}$ is finitary whenever one of the following two conditions is satisfied:

(1) Each algebra $\boldsymbol{A}$ from the quasivariety generated by $\mathbb{K}$ can be regularly embedded into some element of $\mathbb{K}$.

(2) Each finitely subdirectly irreducible algebra $\boldsymbol{A}$ from the variety generated by $\mathbb{K}$ can be regularly embedded into some element of $\mathbb{K}$.

Example 2. In particular, $\models_{\mathbb{K}}$ is finitary for any class $\mathbb{K}$ satisfying one of the following conditions:

- $\mathbb{K}$ is the class of complete algebras of a variety $\mathbb{V}$ of $\mathrm{FL}_{\mathrm{e}}$-algebras admitting regular completions (i.e., such that each $\boldsymbol{A} \in \mathbb{V}$ can be regularly embedded into a complete member of $\mathbb{V}$ ). This is the case, e.g., if $\mathbb{V}$ is axiomatized relative to the class of $\mathrm{FL}_{\mathrm{e}}$-algebras by so-called $\mathrm{N}_{2}$-identities (see [7]), in particular, if $\mathbb{K}$ is the class of all complete $\mathrm{FL}_{\mathrm{e}}$-algebras or complete Heyting algebras.

- $\mathbb{K}$ is the class of complete chains of a variety $\mathbb{V}$ of $F_{\mathrm{e}}$-algebras whose class of chains admits regular completions. This is the case, e.g., if $\mathbb{V}$ is axiomatized relative to the class of integral $\mathrm{FL}_{\mathrm{e}}$-algebras by so-called $\mathrm{P}_{3}$-identities (see [7]), in particular, if $\mathbb{K}$ is the class of all complete Gödel chains or MTL-chains.

Also $\models_{[0,1]_{\mathrm{G}}}$ is known to be finitary. On the other hand, if $\mathbb{K}$ is $\left\{[0,1]_{\mathrm{E}}\right\}$ or the class of all finite MV-algebras, we obtain well-known cases where $\models_{\mathbb{K}}$ is non-finitary.

\footnotetext{
${ }^{5}$ I.e., embedded by a function preserving all suprema and infima of all subsets of $A$ which exist in $\boldsymbol{A}$.
} 


\subsection{Strong and Weak Quantifiers}

Let us fix again a lattice-oriented signature $\mathcal{L}$ and a predicate language $\mathcal{P}=\langle\mathbf{P}, \mathbf{F}$, ar $\rangle$. We write $\varphi[\bullet]$ to denote that an $\langle\mathcal{L}, \mathcal{P}\rangle$-formula $\varphi$ contains a specific occurrence of a subformula $\bullet$ and subsequently $\varphi[\chi]$ to denote the result of replacing $\bullet$ in $\varphi$ with another $\langle\mathcal{L}, \mathcal{P}\rangle$-formula $\chi$. We can make use of the polarities of $\mathcal{L}$ to denote that a specific occurrence of a subformula $\bullet$ in a $\langle\mathcal{L}, \mathcal{P}\rangle$-formula $\varphi$ is either positive (written $\varphi[\bullet]^{+}$) or negative (written $\varphi[\bullet]^{-}$). Formally, the occurrence is positive if one of the following conditions holds, and negative otherwise, proceeding inductively:

$$
\begin{aligned}
& -\varphi=\bullet ; \\
& -\varphi=(Q x) \psi \text { and } \psi[\bullet]^{+}, \text {where } Q \text { is either } \forall \text { or } \exists ; \\
& -\varphi=c\left(\varphi_{1}, \ldots, \varphi_{n}\right) \text { and } \varphi_{i}[\bullet]^{+} \text {, for some } i \in\{1, \ldots, n\} \text { such that } p_{c}(i)=+; \\
& -\varphi=c\left(\varphi_{1}, \ldots, \varphi_{n}\right) \text { and } \varphi_{i}[\bullet]^{-} \text {, for some } i \in\{1, \ldots, n\} \text { such that } p_{c}(i)=-
\end{aligned}
$$

These notions extend also to inequations. If $\Phi$ is an inequation $\varphi \leq \psi$, we denote by $\Phi[\bullet]$ that $\bullet$ is an occurrence of a subformula of either $\varphi$ or $\psi$. Then $\Phi[\bullet]^{+}$stands for $\varphi[\bullet]^{-}$or $\psi[\bullet]^{+}$, and $\Phi[\bullet]^{-}$for $\varphi[\bullet]^{+}$or $\psi[\bullet]^{-}$. Note that this definition conforms with the intuition identifying an inequation $\varphi \leq \psi$ with a formula $\varphi \rightarrow \psi$, where $p_{\rightarrow}(1)=-$ and $p_{\rightarrow}(2)=+$.

We call a positive occurrence of a universally quantified subformula $(\forall x) \psi$ in a formula/inequation strong, and a negative occurrence weak. For existentially quantified formulas the definition is dual: negative occurrences of $(\exists x) \psi$ in a formula/inequation are strong, and positive occurrences are weak. We use a superscript $s$ or $w$ as follows to denote that an occurrence of a quantified subformula $(Q x) \psi$ for $Q \in\{\forall, \exists\}$ in a formula $\varphi$ or inequation $\Phi$ is strong or weak, respectively:

$$
\varphi[(Q x) \psi]^{s}, \quad \varphi[(Q x) \psi]^{w}, \quad \Phi[(Q x) \psi]^{s}, \text { and } \Phi[(Q x) \psi]^{w} .
$$

Let us call a formula/inequation $g$-universal if it contains only strong occurrences of quantified subformulas and $g$ existential if it contains only weak occurrences of quantified subformulas. Note that an inequation $\varphi \leq \psi$ is g-universal if $\varphi$ is g-existential and $\psi$ is g-universal, and g-existential if $\varphi$ is g-universal and $\psi$ is g-existential. Quantifier-free formulas are both g-universal and g-existential.

Example 3. Consider the signature $\mathcal{L}_{1}$ from Example 1 and a predicate language $\mathcal{P}$ consisting of three unary predicate symbols $P_{1}, P_{2}$, and $P_{3}$. For the formula

$$
\varphi=P_{1}(x) \rightarrow\left(P_{2}(x) \vee P_{3}(x)\right),
$$

we have $\varphi\left[P_{1}(x)\right]^{-}, \varphi\left[P_{2}(x)\right]^{+}$, and $\varphi\left[P_{3}(x)\right]^{+}$, while for the inequation

$$
\Phi=P_{1}(x) \rightarrow\left(P_{2}(x) \vee P_{3}(x)\right) \leq P_{3}(x),
$$

we have $\Phi\left[P_{1}(x)\right]^{+}, \Phi\left[P_{2}(x)\right]^{-}$, and either $\Phi\left[P_{3}(x)\right]^{+}$or $\Phi\left[P_{3}(x)\right]^{-}$, depending on which occurrence of $P_{3}(x)$ we are referring to. For the formula

$$
\varphi=(\exists x) P_{1}(x) \rightarrow\left((\exists x) P_{2}(x) \vee(\forall x) P_{3}(x)\right),
$$

we have $\varphi\left[(\exists x) P_{1}(x)\right]^{s}, \varphi\left[(\exists x) P_{2}(x)\right]^{w}$, and $\varphi\left[(\forall x) P_{3}(x)\right]^{s}$, while for the inequation

$$
\Phi=(\exists x) P_{1}(x) \rightarrow\left((\exists x) P_{2}(x) \vee(\forall x) P_{3}(x)\right) \preceq(\forall x) P_{3}(x),
$$

we have $\Phi\left[(\exists x) P_{1}(x)\right]^{w}, \Phi\left[(\exists x) P_{2}(x)\right]^{s}, \Phi\left[(\forall x) P_{3}(x)\right]^{s}$ for the occurrence of $P_{3}$ on the left, and $\Phi\left[(\forall x) P_{3}(x)\right]^{w}$ for the occurrence of $P_{3}$ on the right. Also $(\exists x) P_{1}(x) \rightarrow(\forall x) P_{1}(x)$ is a g-universal formula, while $(\exists x) P_{1}(x) \rightarrow(\forall x) P_{1}(x) \leq$ $(\exists x) P_{1}(x)$ is a g-existential inequation.

The following monotonicity properties are now easily established by induction on formula complexity.

Lemma 2. Let $\varphi, \psi$, and $\chi$ be $\langle\mathcal{L}, \mathcal{P}\rangle$-formulas and $\Phi$ an $\langle\mathcal{L}, \mathcal{P}\rangle$-inequation.

(a) If $\varphi[\bullet]^{+}$, then $\{\psi \leq \chi\} \models_{\mathbb{K}} \varphi[\psi] \leq \varphi[\chi]$. 
(b) If $\varphi[\bullet]^{-}$, then $\{\psi \leq \chi\} \models_{\mathbb{K}} \varphi[\chi] \leq \varphi[\psi]$.

(c) If $\Phi[\bullet]^{+}$, then $\{\Phi[\psi], \psi \leq \chi\} \models_{\mathbb{K}} \Phi[\chi]$.

(d) If $\Phi[\bullet]^{-}$, then $\{\Phi[\chi], \psi \leq \chi\} \vDash_{\mathbb{K}} \Phi[\psi]$.

Proof. We prove (a) and (b) jointly by induction on the definitions of $\varphi[\bullet]^{+}$and $\varphi[\bullet]^{-}$, noting that the base case where $\varphi=\bullet$ is immediate.

We prove the induction step for $\varphi=c\left(\varphi_{1}, \ldots, \varphi_{n}\right)$ for the case that $\varphi_{i}[\bullet]^{+}$and $p_{c}(i)=+$, other cases being very similar. Let $\mathfrak{M}=\langle\boldsymbol{A}, \mathbf{M}\rangle$ be a $\mathbb{K}$-model of $\psi \leq \chi$ and let $\mathrm{v}$ be an $\mathfrak{M}$-evaluation. By the induction hypothesis, $\left\|\varphi_{i}[\psi]\right\|_{\mathrm{v}}^{\mathfrak{M}^{\mathrm{M}}} \leq^{A}\left\|\varphi_{i}[\chi]\right\|_{\mathrm{v}}^{\mathfrak{M}}$ and since $c^{A}$ is monotone in the $i$-th argument, $\|\varphi[\psi]\|_{\mathrm{v}}^{\mathfrak{M}} \leq^{A}\|\varphi[\chi]\|_{\mathrm{v}}^{\mathfrak{M}^{\mathrm{N}}}$.

We prove the induction step for $\varphi=(Q x) \delta$ for the case that $Q=\forall$ and $\delta[\bullet]^{+}$, other cases being very similar. Let $\mathfrak{M}=\langle\boldsymbol{A}, \mathbf{M}\rangle$ be a $\mathbb{K}$-model of $\psi \leq \chi$ and let $\mathrm{v}$ be an $\mathfrak{M}$-evaluation. By the induction hypothesis, $\|\delta[\psi]\|_{\mathrm{v}[\mathrm{x} \rightarrow \mathrm{m}]}^{\mathfrak{M}} \leq^{A}$ $\|\delta[\chi]\|_{\mathrm{v}[\mathrm{x} \rightarrow \mathrm{m}]}^{\mathfrak{M}}$ for each $m \in M$. Hence

$$
\|(\forall x) \delta[\psi]\|_{\mathrm{v}}^{\mathfrak{M}}=\inf _{m \in M}\|\delta[\psi]\|_{\mathrm{v}[\mathrm{x} \rightarrow \mathrm{m}]}^{\mathfrak{M}} \leq^{A} \inf _{m \in M}\|\delta[\chi]\|_{\mathrm{v}[\mathrm{x} \rightarrow \mathrm{m}]}^{\mathfrak{M}}=\|(\forall x) \delta[\chi]\|_{\mathrm{v}}^{\mathfrak{M}} .
$$

Finally we prove part (c), noting that the proof of part (d) is analogous. Assume that $\Phi=\alpha \leq \beta$ and $\alpha[\bullet]^{-}$, the case that $\alpha[\bullet]^{+}$being very similar. By part (b), $\{\psi \leq \chi\} \models_{\mathbb{K}} \alpha[\chi] \leq \alpha[\psi]$, and by Lemma $1,\{\alpha[\psi] \leq \beta, \alpha[\chi] \leq \alpha[\psi]\} \models_{\mathbb{K}}$ $\alpha[\chi] \leq \beta$. Hence, by transitivity, $\{\alpha[\psi] \leq \beta, \psi \leq \chi\} \models_{\mathbb{K}} \alpha[\chi] \leq \beta$.

In classical logic, the satisfaction of universal formulas is preserved under taking substructures of a model. Here we obtain similar preservation properties for g-universal and g-existential formulas in lattice-valued logics. Let us call an $\langle\mathcal{L}, \mathcal{P}\rangle$-structure $\mathfrak{M}^{\prime}=\left\langle\boldsymbol{A}, \mathbf{M}^{\prime}\right\rangle$ a substructure of an $\langle\mathcal{L}, \mathcal{P}\rangle$-structure $\mathfrak{M}=\langle\boldsymbol{A}, \mathbf{M}\rangle$ if $M^{\prime} \subseteq M$ and $\circ^{\mathbf{M}^{\prime}}(\vec{a})=\circ^{\mathbf{M}}(\vec{a})$ for each predicate and function symbol $\circ$ of $\mathcal{P}$ and $\vec{a} \in M^{\prime}$.

Lemma 3. Let $\mathfrak{M}^{\prime}$ be a substructure of an $\langle\mathcal{L}, \mathcal{P}\rangle$-structure $\mathfrak{M}$ and let $\mathrm{v}$ be any $\mathfrak{M}^{\prime}$-evaluation.

(a) $\|\chi\|_{\mathrm{v}}^{\mathfrak{M}^{\prime}} \geq^{A}\|\chi\|_{\mathrm{v}}^{\mathfrak{M}}$ for any g-universal $\langle\mathcal{L}, \mathcal{P}\rangle$-formula $\chi$.

(b) $\|\chi\|_{\mathrm{v}}^{\mathfrak{\mathfrak { N } ^ { \prime }}} \leq^{A}\|\chi\|_{\mathrm{v}}^{\mathfrak{M}}$ for any g-existential $\langle\mathcal{L}, \mathcal{P}\rangle$-formula $\chi$.

Proof. We prove both claims jointly by induction on the definition of $\chi$. If $\chi$ is an atomic formula, then clearly $\|\chi\|_{\mathrm{v}}^{\mathfrak{M}{ }^{\prime}}=\|\chi\|_{\mathrm{v}}^{\mathfrak{M}}$. For the induction step we have several cases. If $\chi=(\forall x) \varphi$ for some g-universal formula $\varphi$, then for each $a \in M^{\prime}$,

Hence also

$$
\|\varphi\|_{\mathrm{v}[x \rightarrow a]}^{\mathfrak{M}^{\prime}} \geq^{A}\|\varphi\|_{\mathrm{v}[x \rightarrow a]}^{\mathfrak{M}} \geq^{A} \inf \left\{\|\varphi\|_{\mathrm{v}[x \rightarrow b]}^{\mathfrak{M}} \mid b \in M\right\}=\|(\forall x) \varphi\|_{\mathrm{v}}^{\mathfrak{M}} .
$$

$$
\|(\forall x) \varphi\|_{\mathrm{v}}^{\mathfrak{M}} \leq^{A} \inf \left\{\|\varphi\|_{\mathrm{v}[x \rightarrow a]}^{\mathfrak{\mathfrak { H } ^ { \prime }}} \mid a \in M^{\prime}\right\}=\|(\forall x) \varphi\|_{\mathrm{v}}^{\mathfrak{M}^{\prime}} .
$$

The case where $\chi=(\exists x) \varphi$ for some g-existential formula $\varphi$ is very similar. Note that we cannot have $\chi=(\forall x) \varphi$ for some g-existential formula $\varphi$, since then $\chi$ would be neither g-universal nor g-existential.

Finally assume that $\chi=c\left(\varphi_{1}, \ldots, \varphi_{n}\right)$ for some $c \in C_{\mathcal{L}}$ and $\chi$ is g-existential. Then $\varphi_{i}$ has to be g-existential if $p_{c}(i)=+$ and g-universal otherwise. By the induction hypothesis,

$$
\begin{array}{ll}
\left\|\varphi_{i}\right\|_{\mathrm{v}}^{\mathfrak{N}^{\prime}} \geq^{A}\left\|\varphi_{i}\right\|_{\mathrm{v}}^{\mathfrak{M}} & \text { if } p_{c}(i)=- \\
\left\|\varphi_{i}\right\|_{\mathrm{v}}^{\mathfrak{M}^{\prime}} \leq^{A}\left\|\varphi_{i}\right\|_{\mathrm{v}}^{\mathfrak{M}} & \text { if } p_{c}(i)=+.
\end{array}
$$

By the monotonicity of $c^{A}$,

$$
\|\chi\|_{\mathrm{v}}^{\mathfrak{M}^{\prime}}=c^{A}\left(\left\|\varphi_{1}\right\|_{\mathrm{v}}^{\mathfrak{N}^{\prime}}, \ldots,\left\|\varphi_{n}\right\|_{\mathrm{v}}^{\mathfrak{M}^{\prime}}\right) \leq^{A} c^{A}\left(\left\|\varphi_{1}\right\|_{\mathrm{v}}^{\mathfrak{M}}, \ldots,\left\|\varphi_{n}\right\|_{\mathrm{v}}^{\mathfrak{M}}\right)=\|\chi\|_{\mathrm{v}}^{\mathfrak{M}} .
$$

The case where $\chi$ is g-universal is analogous.

Proposition 2. Let $\mathfrak{M}^{\prime}$ be a substructure of an $\langle\mathcal{L}, \mathcal{P}\rangle$-structure $\mathfrak{M}$.

(a) $\mathfrak{M} \vDash \Phi$ implies $\mathfrak{M}^{\prime} \vDash \Phi$ for any g-universal inequation $\Phi$. 
(b) $\mathfrak{M}^{\prime} \models \Phi$ implies $\mathfrak{M} \vDash \Phi$ for any g-existential sentential inequation $\Phi$.

(c) $\mathfrak{M} \vDash \Phi$ if and only if $\mathfrak{M}^{\prime} \vDash \Phi$ for any quantifier-free sentential inequation $\Phi$.

Proof. Let $\Phi=\varphi \leq \psi$. For (a), $\varphi$ must be a g-existential formula and $\psi$ a g-universal formula. Assume that $\mathfrak{M} \vDash \Phi$. For each $\mathfrak{M}^{\prime}$-evaluation $\mathrm{v}$, by Lemma 3 .

$$
\|\varphi\|_{\mathrm{v}}^{\mathfrak{M}^{\prime}} \leq^{A}\|\varphi\|_{\mathrm{v}}^{\mathfrak{M}} \leq^{A}\|\psi\|_{\mathrm{v}}^{\mathfrak{M}} \leq^{A}\|\psi\|_{\mathrm{v}}^{\mathfrak{M}^{\prime}} .
$$

That is, $\mathfrak{M}^{\prime}=\Phi$.

For (b), $\psi$ must be a g-existential sentence and $\varphi$ a g-universal sentence. Assume that $\mathfrak{M}^{\prime} \vDash \Phi$. For each $\mathfrak{M}^{\prime}-$ evaluation v, by Lemma 3

$$
\|\varphi\|_{\mathrm{v}}^{\mathfrak{M}} \leq^{A}\|\varphi\|_{\mathrm{v}}^{\mathfrak{M}^{\prime}} \leq^{A}\|\psi\|_{\mathrm{v}}^{\mathfrak{M}^{\prime}} \leq^{A}\|\psi\|_{\mathrm{v}}^{\mathfrak{M}}
$$

Since $\varphi$ and $\psi$ are sentences, this entails $\mathfrak{M} \vDash \Phi$.

Part (c) then follows immediately from (a) and (b).

\subsection{Predicate Substitutions}

In first-order classical logic, consequence is easily reduced to the satisfiability of a theory, but in lattice-valued logics, this is in general no longer the case. Nevertheless, consequence can still be simplified so that the right hand side is an inequation between atomic formulas. This step is established using the notion of a predicate substitution.

Let $\mathcal{L}$ be a lattice-oriented signature and let $\mathcal{P}=\langle\mathbf{P}, \mathbf{F}$, ar $\rangle$ be a predicate language. A predicate substitution (for $\mathcal{P}$ ) is a mapping $\sigma$ assigning to each $n$-ary predicate symbol $P \in \mathbf{P}$ an $\langle\mathcal{L}, \mathcal{P}\rangle$-formula $\sigma(P)$ with (at most) $n$ free variables. Given an arbitrary $\langle\mathcal{L}, \mathcal{P}\rangle$-formula $\varphi$, the $\langle\mathcal{L}, \mathcal{P}\rangle$-formula $\sigma(\varphi)$ is the result of substituting in $\varphi$ each occurrence of an atomic formula $P(\vec{t})$ with the $\langle\mathcal{L}, \mathcal{P}\rangle$-formula $\sigma(P)(\vec{t})$. We also denote by $\sigma(T)$ the result of applying $\sigma$ to all formulas occurring in the inequations of an $\langle\mathcal{L}, \mathcal{P}\rangle$-theory $T$.

Lemma 4. For any $\langle\mathcal{L}, \mathcal{P}\rangle$-theory $T \cup\{\varphi \leq \psi\}$ and predicate substitution $\sigma$,

$$
T \vDash_{\mathbb{K}} \varphi \leq \psi \quad \Longrightarrow \quad \sigma(T) \vDash_{\mathbb{K}} \sigma(\varphi) \leq \sigma(\psi) .
$$

Moreover, the converse direction holds when the only predicates in $T \cup\{\varphi \leq \psi\}$ are propositional atoms and these are mapped by $\sigma$ to distinct closed atomic formulas.

Proof. We proceed by contraposition. If $\sigma(T) \forall_{\mathbb{K}} \sigma(\varphi) \leq \sigma(\psi)$, then there is a model $\mathfrak{M}^{\prime}$ of $\sigma(T)$ such that $\mathfrak{M}^{\prime} \not \neq$ $\sigma(\varphi) \leq \sigma(\psi)$. We construct a model $\mathfrak{M}$ with the same domain as $\mathfrak{M}^{\prime}$ where $f^{\mathfrak{M}}=f^{\mathfrak{M}^{\prime}}$ for each $f \in \mathcal{P}$ and $P^{\mathfrak{M}}(\vec{a})=$ $\|\sigma(P)(\vec{a})\|^{\mathfrak{M}^{\prime}}$ for each $P \in \mathcal{P}$. An easy induction establishes that for every formula $\chi$,

$$
\|\chi(\vec{a})\|^{\mathfrak{M}}=\|\sigma(\chi)(\vec{a})\|^{\mathfrak{M} \mathcal{N}^{\prime}}
$$

But then $\mathfrak{M}$ is a model of $T$ such that $\mathfrak{M} \not \neq \varphi \leq \psi$.

For the converse direction, we may assume without loss of generality that there are no quantifiers in $T \cup\{\varphi \leq \psi\}$ and that all predicates of the language occur in $T \cup\{\varphi \leq \psi\}$. If $T \neq_{\mathbb{K}} \varphi \leq \psi$, then there is a model $\mathfrak{M}=\langle\boldsymbol{A}, \mathbf{M}\rangle$ of $T$ such that $\mathfrak{M} \not \neq \varphi \leq \psi$. We define a model $\mathfrak{M}^{\prime}=\left\langle\boldsymbol{A}, \mathbf{M}^{\prime}\right\rangle$ with the domain consisting of all closed terms, function symbols interpreted in the obvious way, and predicate symbols interpreted for some fixed element $a \in A$ by

$$
P^{\mathfrak{M}^{\prime}}(\vec{t})= \begin{cases}\|Q\|^{\mathfrak{M}} & \text { if } \sigma(Q)=P(\vec{t}) \text { for some propositional atom } Q \in \mathcal{P} \\ a & \text { otherwise }\end{cases}
$$

This definition is sound because predicates are mapped by $\sigma$ to distinct closed atomic formulas. To complete the proof we observe that $\|\sigma(\chi)\|^{\mathfrak{M}^{\prime}}=\|\chi\|^{\mathfrak{M}}$ for each $\chi$ occurring in $T \cup\{\varphi \leq \psi\}$.

Corollary 1. Let $T \cup\{\varphi \leq \psi\}$ be an $\langle\mathcal{L}, \mathcal{P}\rangle$-theory such that the free variables in $\varphi \leq \psi$ are among $\vec{x}$. Then for any predicate symbols $P$ and $Q$ of arity $|\vec{x}|$ not occurring in $T \cup\{\varphi \leq \psi\}$,

$$
T \models_{\mathbb{K}} \varphi \leq \psi \Longleftrightarrow T \cup\{P(\vec{x}) \leq \varphi, \psi \leq Q(\vec{x})\} \models_{\mathbb{K}} P(\vec{x}) \leq Q(\vec{x})
$$


Proof. The left-to-right direction follows from the basic properties of $\leq$. For the right-to-left direction, we suppose that $T \cup\{P(\vec{x}) \leq \varphi, \psi \leq Q(\vec{x})\} \models_{\mathbb{K}} P(\vec{x}) \leq Q(\vec{x})$ and consider a predicate substitution mapping $P$ to $\varphi$ and $Q$ to $\psi$ that acts as the identity on all other predicate symbols. Since $P$ and $Q$ do not occur in $T \cup\{\varphi \leq \psi\}$, the previous lemma yields $T \cup\{\varphi \leq \varphi, \psi \leq \psi\} \models_{\mathbb{K}} \varphi \leq \psi$ and hence $T \models_{\mathbb{K}} \varphi \leq \psi$.

The introduction of new predicate symbols can also be used to reduce g-universal inequations occurring on the left of consequences to finite sets of quantifier-free inequations.

Lemma 5. For any g-universal $\langle\mathcal{L}, \mathcal{P}\rangle$-inequation $\Phi$, there is a predicate language $\mathcal{P}^{\prime} \supseteq \mathcal{P}$ and a finite set $F(\Phi)$ of quantifier-free $\left\langle\mathcal{L}, \mathcal{P}^{\prime}\right\rangle$-inequations such that for any $\langle\mathcal{L}, \mathcal{P}\rangle$-theory $T \cup\{\Psi\}$,

$$
T \cup\{\Phi\} \vDash_{\mathbb{K}} \Psi \Longleftrightarrow T \cup F(\Phi) \vDash_{\mathbb{K}} \Psi \text {. }
$$

In particular, $F(\Phi) \models_{\mathbb{K}} \Phi$.

Proof. We prove the claim by induction on the number of quantifiers in $\Phi=\varphi \leq \psi$. The base case, where $\varphi \leq \psi$ is already a quantifier-free inequation, is immediate; we just let $F(\Phi)=\{\Phi\}$. For the induction step, we distinguish four cases based on whether $\varphi$ or $\psi$ has a universal or an existential subformula. We show only one case, since the other cases are very similar.

Suppose that $\varphi$ has a universal subformula. Since $\varphi \leq \psi$ is a g-universal inequation, it must be the case that $\varphi[(\forall \vec{x}) \chi(\vec{x}, \vec{y})]^{-}$for some quantifier-free formula $\chi(\vec{x}, \vec{y})$. Given a predicate symbol $P_{\chi} \notin \mathcal{P}$ of the appropriate arity (the length of $\vec{y}$ ), we first prove that

$$
T \cup\{\varphi \leq \psi\} \vDash_{\mathbb{K}} \Psi \Longleftrightarrow T \cup\left\{P_{\chi}(\vec{y}) \leq \chi(\vec{x}, \vec{y}), \varphi\left[P_{\chi}(\vec{y})\right] \leq \psi\right\} \vDash_{\mathbb{K}} \Psi .
$$

For the left-to-right direction, recall that $\left\{P_{\chi}(\vec{y}) \leq \chi(\vec{x}, \vec{y})\right\} \models_{\mathbb{K}} P_{\chi}(\vec{y}) \leq(\forall \vec{x}) \chi(\vec{x}, \vec{y})$ and so, by Lemma 2, also

$$
\left\{P_{\chi}(\vec{y}) \leq \chi(\vec{x}, \vec{y})\right\} \models_{\mathbb{K}} \varphi[(\forall \vec{x}) \chi(\vec{x}, \vec{y})] \leq \varphi\left[P_{\chi}(\vec{y})\right] .
$$

Hence $\left\{P_{\chi}(\vec{y}) \leq \chi(\vec{x}, \vec{y}), \varphi\left[P_{\chi}(\vec{y})\right] \leq \psi\right\} \models_{\mathbb{K}} \varphi \leq \psi$, and the conclusion follows.

To prove the converse direction, we apply Lemma 4 using a substitution $\sigma$ that is the identity except for $\sigma\left(P_{\chi}\right)=$ $(\forall \vec{x}) \chi(\vec{x}, \vec{y})$ and obtain $T \cup\{(\forall \vec{x}) \chi(\vec{x}, \vec{y}) \leq \chi(\vec{x}, \vec{y}), \varphi[(\forall \vec{x}) \chi(\vec{x}, \vec{y})] \leq \psi\} \models_{\mathbb{K}} \Psi$. Since $\models_{\mathbb{K}}(\forall \vec{x}) \chi(\vec{x}, \vec{y}) \leq \chi(\vec{x}, \vec{y})$, the claim follows.

Now we can apply the induction hypothesis for the predicate language $\mathcal{P} \cup\left\{P_{\chi}\right\},\left\langle\mathcal{L}, \mathcal{P} \cup\left\{P_{\chi}\right\}\right\rangle$-inequation $\varphi\left[P_{\chi}(\vec{y})\right] \leq$ $\psi$, and $\left\langle\mathcal{L}, \mathcal{P} \cup\left\{P_{\chi}\right\}\right\rangle$-theory $T^{\prime}=T \cup\left\{P_{\chi}(\vec{y}) \leq \chi(\vec{x}, \vec{y}), \Phi\right\}$ to obtain a predicate language $\mathcal{P}^{\prime} \supseteq \mathcal{P} \cup\left\{P_{\chi}\right\}$ and finite set of quantifier-free $\left\langle\mathcal{L}, \mathcal{P}^{\prime}\right\rangle$-inequations $F\left(\varphi\left[P_{\chi}(\vec{y})\right] \leq \psi\right)$ such that

$$
T^{\prime} \cup\left\{\varphi\left[P_{\chi}(\vec{y})\right] \leq \psi\right\} \models_{\mathbb{K}} \Psi \Longleftrightarrow T^{\prime} \cup F\left(\varphi\left[P_{\chi}(\vec{y})\right] \leq \psi\right) \vDash \Psi .
$$

The proof is completed by combining equivalences and defining

$$
F(\varphi \leq \psi)=\left\{P_{\chi}(\vec{y}) \leq \chi(\vec{x}, \vec{y})\right\} \cup F\left(\varphi\left[P_{\chi}(\vec{y})\right] \leq \psi\right) .
$$

Example 4. Consider the signature $\mathcal{L}_{1}$ from Example 1, a predicate language $\mathcal{P}$ consisting of a unary predicate symbol $P_{1}$ and binary predicate symbol $P_{2}$, and the g-universal inequation

$$
\Phi=(\forall x) P_{2}(x, y) \rightarrow P_{1}(y) \preceq(\exists x) P_{1}(x) .
$$

In order to obtain the finite set $F(\Phi)$ of quantifier-free inequations, we replace the quantified subformulas $(\forall x) P_{2}(x, y)$ with a new unary predicate symbol $R_{2}$ and $(\exists x) P_{1}(x)$ with a new propositional atom $R_{1}$. Since $(\forall x) P_{2}(x, y)$ appears negatively on the left of $\Phi$, we include in $F(\Phi)$ the inequation $R_{2}(y) \leq P_{2}(x, y)$, and since $(\exists x) P_{1}(x)$ appears positively on the right, we include $P_{1}(x) \leq R_{1}$. Hence we obtain a predicate language $\mathcal{P}^{\prime}$ extending $\mathcal{P}$ with two new predicate symbols $R_{1}$ and $R_{2}$, and the finite set of quantifier-free $\left\langle\mathcal{L}_{1}, \mathcal{P}^{\prime}\right\rangle$-inequations

$$
F(\Phi)=\left\{R_{2}(y) \leq P_{2}(x, y), P_{1}(x) \preceq R_{1}, R_{2}(y) \rightarrow P_{1}(y) \preceq R_{1}\right\} .
$$

Note finally that using this last lemma and Corollary 1 we obtain that a consequence between g-universal inequations on the left and a g-existential inequation on the right reduces to a consequence with quantifier-free inequations on both sides. 


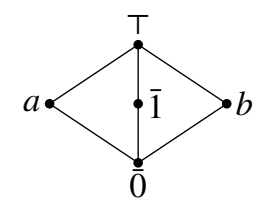

Figure 1: The $\mathrm{FL}_{\mathrm{e}}$-algebra $A$

\section{Skolemization}

We have shown in the last section that consequences between g-universal inequations on the left and a g-existential inequation on the right can be reduced to consequences between quantifier-free inequations. In order to apply this reduction to a more general class of formulas, we consider now Skolemization methods that yield consequences of the desired form by iteratively removing strong occurrences of quantifiers.

As mentioned in the introduction, we cannot expect the usual Skolemization method to be sound in general for first-order lattice-valued logics. Consider, for example, the $\mathrm{FL}_{\mathrm{e}}$-algebra $\boldsymbol{A}=\langle A, \&, \rightarrow, \wedge, \vee, \overline{0}, \overline{1}\rangle$ depicted in Figure 1 with

$$
x \& y= \begin{cases}x \wedge y & \text { if } x, y \in\{0, a, b, \top\} \\ x & \text { if } y=\overline{1} \\ y & \text { if } x=\overline{1} .\end{cases}
$$

Let $\mathfrak{M}=\langle\boldsymbol{A}, \mathbf{M}\rangle$ be a structure for the first-order language with a binary predicate symbol $P$ and object constants $r$ and $s$, where $M=\{r, s\}, r^{\mathbf{M}}=r, s^{\mathbf{M}}=s$, and

$$
P^{\mathbf{M}}(r, r)=\overline{1}, \quad P^{\mathbf{M}}(r, s)=P^{\mathbf{M}}(s, s)=a, \quad P^{\mathbf{M}}(s, r)=b .
$$

It is easily confirmed that

$$
\mathfrak{M} \vDash \overline{1} \leq(\forall x)(\forall z)(P(x, r) \vee P(z, s)) \quad \text { and } \quad \mathfrak{M} \not \overline{1} \leq(\exists y)(\forall x) P(x, y),
$$

and hence

$$
\{\overline{1} \leq(\forall x)(\forall z)(P(x, r) \vee P(z, s))\} \nvdash_{A} \overline{1} \leq(\exists y)(\forall x) P(x, y) .
$$

On the other hand, for any unary function symbol $f$,

$$
\{\overline{1} \leq(\forall x)(\forall z)(P(x, r) \vee P(z, s))\} \models_{A} \overline{1} \leq(\exists y) P(f(y), y) .
$$

Hence "ordinary" Skolemization in this case is not sound. Suppose, however, that we introduce two new unary function symbols $f_{1}$ and $f_{2}$. Then extending the structure $\mathfrak{M}$ with interpretations $f_{1}^{\mathbf{M}}(r)=f_{1}^{\mathbf{M}}(s)=r$ and $f_{2}^{\mathbf{M}}(r)=$ $f_{2}^{\mathbf{M}}(s)=s$, we obtain $\left\|(\exists y)\left(P\left(f_{1}(y), y\right) \wedge P\left(f_{2}(y), y\right)\right)\right\|^{\mathfrak{M}}=a \nsupseteq \overline{1}$ and

$$
\{\overline{1} \leq(\forall x)(\forall z)(P(x, r) \vee P(z, s))\} \not_{A} \overline{1} \leq(\exists y)\left(P\left(f_{1}(y), y\right) \wedge P\left(f_{2}(y), y\right)\right) .
$$

More generally, for any theory $T \cup\{\psi \leq(\exists \bar{y})(\forall x) \varphi(x, \bar{y})\}$ of this language and new function symbols $f_{1}, f_{2}$ of arity $|\bar{y}|$,

$$
T \models_{A} \psi \leq(\exists \bar{y})(\forall x) \varphi(x, \bar{y}) \Longleftrightarrow T \vDash_{A} \psi \leq(\exists \bar{y})\left(\varphi\left(f_{1}(\bar{y}), \bar{y}\right) \wedge \varphi\left(f_{2}(\bar{y}), \bar{y}\right)\right) .
$$

In this section, we relate the soundness of this "parallel Skolemization" method to certain witnessed model properties of the lattice-valued logic. 


\subsection{Parallel Skolemization}

Let us fix again a lattice-oriented signature $\mathcal{L}$ and a predicate language $\mathcal{P}=\langle\mathbf{P}, \mathbf{F}$, ar $\rangle$. Consider an $\langle\mathcal{L}, \mathcal{P}\rangle$-formula $\varphi[(Q x) \psi(x, \vec{y})]$ with $Q \in\{\exists, \forall\}, n \in \mathbb{N}^{+}$, and function symbols $f_{1}, \ldots, f_{n} \notin \mathcal{P}$ of arity $|\vec{y}|$. We obtain the new formula

$$
\varphi\left[\bigvee_{i=1}^{n} \psi\left(f_{i}(\vec{y}), \vec{y}\right)\right] \text { if } Q=\exists \text { and } \varphi\left[\bigwedge_{i=1}^{n} \psi\left(f_{i}(\vec{y}), \vec{y}\right)\right] \text { if } Q=\forall .
$$

This replacement step strictly decreases the finite multiset of depths of occurrences of quantifiers according to the standard multiset well-ordering ${ }^{6}$ Hence repeatedly applying it to leftmost strong occurrences of quantifiers in an arbitrary $\langle\mathcal{L}, \mathcal{P}\rangle$-formula $\varphi$ results in a unique (up to renaming of function symbols) $\left\langle\mathcal{L}, \mathcal{P}^{\prime}\right\rangle$-formula $\operatorname{sk}_{n}^{\exists}(\varphi)$ for some extension $\mathcal{P}^{\prime}$ of $\mathcal{P}$ that contains only weak occurrences of quantifiers. Similarly, let $\operatorname{sk}_{n}^{\forall}(\varphi)$ be the result of applying this process repeatedly to leftmost weak occurrences of quantifiers in $\varphi$. Note that $\operatorname{sk}_{n}^{\exists}(\varphi)$ is a g-existential formula and $\operatorname{sk}_{n}^{\forall}(\varphi)$ is a g-universal formula; hence, we speak of the existential and universal $n$-Skolem form of $\varphi$.

The existential and universal $n$-Skolem forms of an inequation $\varphi \leq \psi$ are defined analogously, yielding

$$
\operatorname{sk}_{n}^{\exists}(\varphi \leq \psi)=\operatorname{sk}_{n}^{\forall}(\varphi) \leq \operatorname{sk}_{n}^{\exists}(\psi) \text { and } \operatorname{sk}_{n}^{\forall}(\varphi \leq \psi)=\operatorname{sk}_{n}^{\exists}(\varphi) \leq \operatorname{sk}_{n}^{\forall}(\psi),
$$

where the new function symbols appearing in $\operatorname{sk}_{n}^{\exists}(\varphi)$ and $\operatorname{sk}_{n}^{\forall}(\psi)$ are distinct.

Remark 2. Note that we could define (potentially) smaller $n$-Skolem forms by dealing with multiple occurrences of a quantifier simultaneously, that is, by considering formulas $\varphi[(Q \vec{x}) \psi(\vec{x}, \vec{y})]$ for $Q \in\{\exists, \forall\}$, where $Q \vec{x}$ is a (maximal within the formula) string $Q x_{1} Q x_{2} \ldots Q x_{n}$.

Example 5. Let us consider the signature $\mathcal{L}_{1}$ from Example 1 and a predicate language $\mathcal{P}$ consisting of two binary predicate symbols $P_{1}$ and $P_{2}$. Let $\varphi$ be the sentence

$$
(\forall x)\left((\exists y) P_{1}(x, y) \rightarrow(\exists z) P_{2}(x, z)\right) .
$$

Taking $n=1$, the above process leads to

$$
\begin{aligned}
\operatorname{sk}_{1}^{\exists}(\varphi) & =P_{1}(c, d) \rightarrow(\exists z) P_{2}(c, z), \\
\operatorname{sk}_{1}^{\forall}(\varphi) & =(\forall x)\left((\exists y) P_{1}(x, y) \rightarrow P_{2}(x, g(x))\right) .
\end{aligned}
$$

On the other hand, for $n=2$, it produces

$$
\begin{aligned}
& \operatorname{sk}_{2}^{\exists}(\varphi)=\left(\left(P_{1}\left(c_{1}, d_{1}^{1}\right) \vee P_{1}\left(c_{1}, d_{2}^{1}\right)\right) \rightarrow(\exists z) P_{2}\left(c_{1}, z\right)\right) \wedge\left(\left(P_{1}\left(c_{2}, d_{1}^{2}\right) \vee P_{1}\left(c_{2}, d_{2}^{2}\right)\right) \rightarrow(\exists z) P_{2}\left(c_{2}, z\right)\right), \\
& \operatorname{sk}_{2}^{\forall}(\varphi)=(\forall x)\left((\exists y) P_{1}(x, y) \rightarrow\left(P_{2}\left(x, g_{1}(x)\right) \vee P_{2}\left(x, g_{2}(x)\right)\right)\right) .
\end{aligned}
$$

Consider now the inequation

$$
\Phi=(\forall x)\left((\exists y) P_{1}(x, y) \rightarrow(\exists z) P_{2}(x, z)\right) \preceq(\forall x)(\exists y) P_{1}(x, y) .
$$

Applying the above process for $n=1$ we obtain the following inequations

$$
\begin{aligned}
& \operatorname{sk}_{1}^{\exists}(\Phi)=(\forall x)\left((\exists y) P_{1}(x, y) \rightarrow P_{2}(x, g(x))\right) \leq(\exists y) P_{1}(c, y), \\
& \operatorname{sk}_{1}^{\forall}(\Phi)=P_{1}(c, d) \rightarrow(\exists z) P_{2}(c, z) \leq(\forall x) P_{1}(x, g(x)) .
\end{aligned}
$$

\footnotetext{
${ }^{6}$ A finite multiset over a set $S$ is an ordered pair $\langle S, f\rangle$, where $f$ is a function $f: S \rightarrow \mathbb{N}$ and $\{x \in S \mid f(x)>0\}$ is finite. If $\leq$ is a well-ordering of $S$, then

$$
\langle S, f\rangle \leq_{m}\langle S, g\rangle \quad: \Longleftrightarrow \forall x \in S(f(x)>g(x) \Longrightarrow \exists y \in S(y>x \text { and } g(y)>f(y)))
$$

is a well-ordering on the set of all finite multisets over $S$, known as the Dershowitz-Manna ordering [12].
} 
Let us now fix $\mathbb{K}$ to be any class of complete $\mathcal{L}$-lattices.

Lemma 6. For any $\langle\mathcal{L}, \mathcal{P}\rangle$-formula $\varphi$ and $\langle\mathcal{L}, \mathcal{P}\rangle$-inequation $\Phi$,

(a) $\models_{\mathbb{K}} \operatorname{sk}_{n}^{\forall}(\varphi) \leq \varphi$ and $\models_{\mathbb{K}} \varphi \leq \operatorname{sk}_{n}^{\exists}(\varphi)$;

(b) $\operatorname{sk}_{n}^{\forall}(\Phi) \models_{\mathbb{K}} \Phi$ and $\Phi \models_{\mathbb{K}} \operatorname{sk}_{n}^{\exists}(\Phi)$.

Proof. We prove only $\models_{\mathbb{K}} \operatorname{sk}_{n}^{\forall}(\varphi) \leq \varphi$ as the other properties follow similarly. Suppose that $\varphi[(Q x) \psi(x, \vec{y})]^{w}$, i.e., $\varphi[(\exists x) \psi(x, \vec{y})]^{+}$or $\varphi[(\forall x) \psi(x, \vec{y})]^{-}$. Clearly

$$
\models_{\mathbb{K}} \bigvee_{i=1}^{n} \psi\left(f_{i}(\vec{y}), \vec{y}\right) \leq(\exists x) \psi(x, \vec{y}) \quad \text { and } \quad \models_{\mathbb{K}}(\forall x) \psi(x, \vec{y}) \leq \bigwedge_{i=1}^{n} \psi\left(f_{i}(\vec{y}), \vec{y}\right),
$$

so using Lemma 2 we obtain

$$
\models_{\mathbb{K}} \varphi\left[\bigvee_{i=1}^{n} \psi\left(f_{i}(\vec{y}), \vec{y}\right)\right] \leq \varphi[(\exists x) \psi(x, \vec{y})] \quad \text { or } \quad \models_{\mathbb{K}} \varphi\left[\bigwedge_{i=1}^{n} \psi\left(f_{i}(\vec{y}), \vec{y}\right)\right] \leq \varphi[(\forall x) \psi(x, \vec{y})]
$$

Applying this process repeatedly to leftmost weak occurrences of quantifiers in $\varphi$, we obtain $\models_{\mathbb{K}} \operatorname{sk}_{n}^{\forall}(\varphi) \leq \varphi$ by transitivity.

We say that $\models_{\mathbb{K}}$ admits parallel Skolemization of degree $n$ if for any predicate language $\mathcal{P}$ and $\langle\mathcal{L}, \mathcal{P}\rangle$-theory $T \cup\{\Phi, \Psi\}$,

$$
T \cup\{\Phi\} \vDash_{\mathbb{K}} \Psi \Longleftrightarrow T \cup\left\{\operatorname{sk}_{n}^{\forall}(\Phi)\right\} \vDash_{\mathbb{K}} \Psi .
$$

Note that our definition is formulated for premises only; in a subsequent lemma we show that this property entails parallel Skolemization of degree $n$ also for conclusions. The question whether the converse implication holds is open, but we conjecture that this is not the case. Note also that although we formulate the property as an equivalence, the right-to-left direction is trivial due to the previous lemma.

Remark 3. Parallel Skolemization of degree $n$ for $\models_{\mathbb{K}}$ may also be understood as meaning that $T \cup\left\{\operatorname{sk}_{n}^{\forall}(\Phi)\right\}$ is a conservative extension of $T \cup\{\Phi\}$.

Lemma 7. If $\models_{\mathbb{K}}$ admits parallel Skolemization of degree $n$, then for any predicate language $\mathcal{P}$ and $\langle\mathcal{L}, \mathcal{P}\rangle$-theory $T \cup\{\Phi\}$,

$$
T \models_{\mathbb{K}} \Phi \Longleftrightarrow T \models_{\mathbb{K}} \operatorname{sk}_{n}^{\exists}(\Phi)
$$

Proof. Let $\Phi=\varphi \leq \psi$ and let $P, Q$ be predicate symbols of the appropriate arity not occurring in $T \cup\{\varphi \leq \psi\}$. We obtain that

$$
\begin{aligned}
T \models_{\mathbb{K}} \varphi \leq \psi & \Leftrightarrow T \cup\{P(\vec{x}) \leq \varphi, \psi \leq Q(\vec{x})\} \models_{\mathbb{K}} P(\vec{x}) \leq Q(\vec{x}) \\
& \Leftrightarrow T \cup\left\{\operatorname{sk}_{n}^{\forall}(P(\vec{x}) \leq \varphi), \operatorname{sk}_{n}^{\forall}(\psi \leq Q(\vec{x}))\right\} \models_{\mathbb{K}} P(\vec{x}) \leq Q(\vec{x}) \\
& \Leftrightarrow T \cup\left\{\operatorname{sk}_{n}^{\exists}(P(\vec{x})) \leq \operatorname{sk}_{n}^{\forall}(\varphi), \operatorname{sk}_{n}^{\exists}(\psi) \leq \operatorname{sk}_{n}^{\forall}(Q(\vec{x}))\right\} \models_{\mathbb{K}} P(\vec{x}) \leq Q(\vec{x}) \\
& \Leftrightarrow T \cup\left\{P(\vec{x}) \leq \operatorname{sk}_{n}^{\forall}(\varphi), \operatorname{sk}_{n}^{\exists}(\psi) \leq Q(\vec{x})\right\} \models_{\mathbb{K}} P(\vec{x}) \leq Q(\vec{x}) \\
& \Leftrightarrow T \models_{\mathbb{K}} \operatorname{sk}_{n}^{\forall}(\varphi) \leq \operatorname{sk}_{n}^{\exists}(\psi) . \\
& \Leftrightarrow T \models_{\mathbb{K}} \operatorname{sk}_{n}^{\exists}(\varphi \leq \psi) .
\end{aligned}
$$

Equivalences (1) and (5) follow from Corollary 1, (2) follows from the assumption that $\models_{\mathbb{K}}$ admits parallel Skolemization of degree $n$, and (3), (4), and (6), follow from the definitions of $\operatorname{sk}_{n}^{\exists}(\cdot)$ and $\operatorname{sk}_{n}^{\forall}(\cdot)$. 


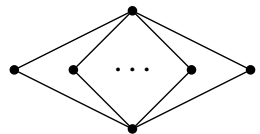

(a)

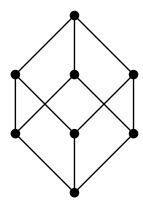

(b)

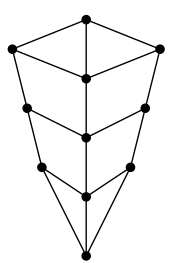

(c)

Figure 2: Examples of 2-compact and 3-compact systems

\subsection{Witnessed Model Properties}

We consider now consequence relations $\models_{\mathbb{K}}$ that admit parallel Skolemization of some fixed degree. As we will see in Theorem 11, the key prerequisite is the completeness of $\models_{\mathbb{K}}$ with respect to models based on classes of $\mathcal{L}$-lattices exhibiting some degree of "compactness".

Let $\boldsymbol{L}$ be an $\mathcal{L}$-lattice and $\mathcal{X} \subseteq \mathcal{P}(L)$. We say that $\mathcal{X}$ is $n$-compact for some $n \in \mathbb{N}^{+}$if for each $A \in \mathcal{X}$ and $b \in L$,

$$
\begin{aligned}
& \wedge A \leq b \quad \Longrightarrow \quad a_{1} \wedge \ldots \wedge a_{n} \leq b \text { for some } a_{1}, \ldots, a_{n} \in A \text {, } \\
& \vee A \geq b \quad \Longrightarrow \quad a_{1} \vee \ldots \vee a_{n} \geq b \text { for some } a_{1}, \ldots, a_{n} \in A \text {. }
\end{aligned}
$$

Equivalently, for each $A \in \mathcal{X}$, there exist $a_{1}, \ldots, a_{n}, b_{1}, \ldots, b_{n} \in A$ such that $\wedge A=a_{1} \wedge \ldots \wedge a_{n}$ and $\vee A=b_{1} \vee \ldots \vee b_{n}$. Obviously, if $\mathcal{X}$ is $n$-compact then it is also $m$-compact for any $m \in \mathbb{N}^{+}$greater than $n$.

Example 6. It is easily seen that if (the underlying lattice of) $\boldsymbol{L}$ has height (i.e, the cardinality of a maximal chain in $\boldsymbol{L}$ ) smaller than $n+1$, then any $\mathcal{X} \subseteq \mathcal{P}(L)$ is $n$-compact. If $\boldsymbol{L}$ contains no infinite chain and has width (i.e, the cardinality of a maximal anti-chain in $\boldsymbol{L}$ ) smaller than $m$, then any $\mathcal{X} \subseteq \mathcal{P}(L)$ is $m$-compact. For example, the powerset of a lattice, as depicted in Figure 2(a), that consists of a (finite or infinite) set of incomparable elements together with a top element and a bottom element, is 2-compact (but not 1-compact). The powerset of the lattice in Figure 2 (b), which may also be generalized by repeating many times the internal elements, is 3-compact (but not 2-compact). On the other hand, the powerset of the lattice in Figure 2(c) is 2-compact (but not 1-compact).

In order for a consequence relation $\models_{\mathbb{K}}$ to admit parallel Skolemization of degree $n$, it is not mandatory that all sets of subsets of the $\mathcal{L}$-lattices in $\mathbb{K}$ be $n$-compact, only that the set of definable sets of elements in a given $\langle\mathcal{L}, \mathcal{P}\rangle$-structure have this property. Let us call an $\langle\mathcal{L}, \mathcal{P}\rangle$-structure $\mathcal{S}=\langle\boldsymbol{A}, \mathbf{S}\rangle n$-witnessed if the following system is $n$-compact:

$$
\left\{\left\{\|\varphi(b, \vec{a})\|^{\subseteq} \mid b \in S\right\} \mid \varphi(x, \vec{y}) \text { an }\langle\mathcal{L}, \mathcal{P}\rangle \text {-formula and } \vec{a} \subseteq S\right\} .
$$

The consequence relation $\models_{\mathbb{K}}$ has the $n$-witnessed model property if for any $\langle\mathcal{L}, \mathcal{P}\rangle$-theory $T \cup\{\Phi\}$,

$$
T \models_{\mathbb{K}} \Phi \Longleftrightarrow \text { each } n \text {-witnessed model } \mathfrak{M} \text { of } T \text { is a model of } \Phi \text {. }
$$

Note that this notion generalizes the (1-)witnessed model property introduced by Hájek in [19] (see also [6, 10]).

Example 7. Suppose that $\mathbb{K}$ is a class of $\mathrm{FL}_{\mathrm{e}}$-algebras (see Example 1) whose underlying lattices either have height bounded by some fixed $n+1$, or contain no infinite chain and have width bounded by some fixed $n$ (see Example 6). Then $\models_{\mathbb{K}}$ has the $n$-witnessed model property.

Example 8. Let us emphasize that it is not necessary for parallel Skolemization that all sets of subsets of $\mathcal{L}$-lattices in the class $\mathbb{K}$ are $n$-compact. Suppose, for example, that $\mathbb{K}=\left\{[0,1]_{\mathrm{E}}\right\}$ (see Example 1 ). The powerset of $[0,1]$ is clearly not $n$-compact for any $n$. However, $\models_{\mathbb{K}}$ has the 1-witnessed model property, as shown by Caicedo [6], correcting an earlier proof of Hájek [19].

We turn our attention now to the relationship between the $n$-witnessed model property and parallel Skolemization of degree $n$. We begin with a crucial lemma which can be understood as a characterization of "one step" Skolemization. 
Lemma 8. Suppose that $\models_{\mathbb{K}}$ has the n-witnessed model property. Then for any $\langle\mathcal{L}, \mathcal{P}\rangle$-theory $T \cup\{\Phi, \Psi\}$ and function symbols $f_{1}, \ldots, f_{n} \notin \mathcal{P}$ of arity $|\vec{y}|$,

$$
\begin{aligned}
T \cup\{\Phi\} \models_{\mathbb{K}} \Psi \Longleftrightarrow T \cup\left\{\Phi\left[\bigvee_{i=1}^{n} \chi\left(f_{i}(y), \vec{y}\right)\right]\right\} \models_{\mathbb{K}} \Psi & \text { if } \Phi[(\exists x) \chi(x, \vec{y})]^{+} \\
T \cup\{\Phi\} \models_{\mathbb{K}} \Psi \Longleftrightarrow T \cup\left\{\Phi\left[\bigwedge_{i=1}^{n} \chi\left(f_{i}(y), \vec{y}\right)\right]\right\} \models_{\mathbb{K}} \Psi & \text { if } \Phi[(\forall x) \chi(x, \vec{y})]^{-} .
\end{aligned}
$$

Proof. The left-to-right directions for both claims follow from Lemma 2 (see also the proof of Lemma 6). For the other direction, assume that $\Phi=\varphi \leq \psi$ and $\psi[(\forall x) \chi(x, \vec{y})]^{-}$. Note that the other cases $\left(\psi[(\exists x) \chi(x, \vec{y})]^{+}, \varphi[(\exists x) \chi(x, \vec{y})]^{-}\right.$, and $\left.\varphi[(\forall x) \chi(x, \vec{y})]^{+}\right)$are very similar.

We proceed contrapositively. Suppose that $T \cup\{\Phi\} \forall_{\mathbb{K}} \Psi$, so there is an $n$-witnessed model $\mathfrak{M}=\langle\boldsymbol{A}, \mathbf{M}\rangle$ of $T \cup\{\Phi\}$ such that $\mathfrak{M} \forall_{\mathbb{K}} \Psi$. Because $\mathfrak{M}$ is $n$-witnessed, for each $\vec{m} \subseteq M$, there are $u_{1}^{\vec{m}}, \ldots, u_{n}^{\vec{m}} \in M$ such that

$$
\|(\forall x) \chi(x, \vec{m})\|^{\mathfrak{M}}=\left\|\chi\left(u_{1}^{\vec{m}}, \vec{m}\right)\right\|^{\mathfrak{M}} \wedge \ldots \wedge\left\|\chi\left(u_{n}^{\vec{m}}, \vec{m}\right)\right\|^{\mathfrak{M}} .
$$

Using the axiom of choice, we define $f_{i}(\vec{m})=u_{i}^{\vec{m}}$ for each $i \in\{1, \ldots, n\}$. Then $\mathfrak{M}$, with these new interpretations, is a model of $T \cup\left\{\varphi \leq \psi\left[\bigwedge_{i=1}^{n} \chi\left(f_{i}(\vec{y}), \vec{y}\right)\right]\right\}$ and not of $\Psi$.

Theorem 1. If $\models_{\mathbb{K}}$ has the n-witnessed model property, then $\models_{\mathbb{K}}$ admits parallel Skolemization of degree n. Moreover, the converse implication also holds whenever $\models_{\mathbb{K}}$ is finitary.

Proof. Suppose that $\models_{\mathbb{K}}$ has the $n$-witnessed model property. Parallel Skolemization of degree $n$ follows from Lemma 8 and an induction on the multiset of depths of quantifier occurrences according to the Derschowitz-Manna ordering (see Footnote 6).

Let us prove the converse. Suppose that $\models_{\mathbb{K}}$ is finitary and admits parallel Skolemization of degree $n$. First we establish the following:

Claim. For each $\langle\mathcal{L}, \mathcal{P}\rangle$-theory $T \cup\{\Psi\}$ such that $T \forall_{\mathbb{K}} \Psi$, there exist a language $\mathcal{P}^{\prime} \supseteq \mathcal{P}$ and a $\left\langle\mathcal{L}, \mathcal{P}^{\prime}\right\rangle$-theory $T^{\prime} \supseteq T$ such that $T^{\prime} \forall_{\mathbb{K}} \Psi$ and, for each $\langle\mathcal{L}, \mathcal{P}\rangle$-formula $(Q x) \psi(x, \vec{y})$,

$$
T^{\prime} \vDash_{\mathbb{K}}(Q x) \psi(x, \vec{y}) \approx \bigcirc_{i=1}^{n} \psi\left(f_{i}^{\psi}(\vec{y}), \vec{y}\right),
$$

where $\bigcirc=\left\{\begin{array}{ll}\vee & \text { if } Q=\exists \\ \wedge & \text { if } Q=\forall\end{array}\right.$ and $f_{1}^{\psi}, \ldots, f_{n}^{\psi}$ are function symbols from $\mathcal{P}^{\prime} \backslash \mathcal{P}$.

Proof of the claim. Let $\varphi_{0}, \varphi_{1}, \ldots$ be an enumeration of all $\langle\mathcal{L}, \mathcal{P}\rangle$-formulas of the form $(\forall x) \psi(x, \vec{y})$ or $(\exists x) \psi(x, \vec{y})$ (recalling that $\mathcal{P}$ is always a countable language). We construct increasing sequences of languages $\mathcal{P}_{i}$ and $\left\langle\mathcal{L}, \mathcal{P}_{i}\right\rangle-$ theories $T_{i}$ such that $T_{i} \forall_{\mathbb{K}} \Psi$. Let $T_{0}=T$ and $\mathcal{P}_{0}=\mathcal{P}$. If $\varphi_{j}$ has the form $(\forall x) \psi(x, \vec{y})$, then, since $\models_{\mathbb{K}}$ admits parallel Skolemization of degree $n$,

$$
\begin{aligned}
T_{j} \models_{\mathbb{K}} \Psi & \Longleftrightarrow T_{j} \cup\{(\forall x) \psi(x, \vec{y}) \leq(\forall x) \psi(x, \vec{y})\} \models_{\mathbb{K}} \Psi \\
& \Longleftrightarrow T_{j} \cup\left\{\operatorname{sk}_{n}^{\forall}((\forall x) \psi(x, \vec{y}) \leq(\forall x) \psi(x, \vec{y}))\right\} \models_{\mathbb{K}} \Psi \\
& \Longleftrightarrow T_{j} \cup\left\{\operatorname{sk}_{n}^{\forall}\left(\bigwedge_{i=1}^{n} \psi\left(f_{i}^{\psi}(\vec{y}), \vec{y}\right) \leq(\forall x) \psi(x, \vec{y})\right)\right\} \models_{\mathbb{K}} \Psi \\
& \Longleftrightarrow T_{j} \cup\left\{\bigwedge_{i=1}^{n} \psi\left(f_{i}^{\psi}(\vec{y}), \vec{y}\right) \leq(\forall x) \psi(x, \vec{y})\right\} \models_{\mathbb{K}} \Psi .
\end{aligned}
$$

We define $\mathcal{P}_{j+1}$ as the extension of $\mathcal{P}_{j}$ with the function symbols $f_{1}^{\psi}, \ldots, f_{n}^{\psi}$ and let

$$
T_{j+1}=T_{j} \cup\left\{\bigwedge_{i=1}^{n} \psi\left(f_{i}^{\psi}(\vec{y}), \vec{y}\right) \leq(\forall x) \psi(x, \vec{y})\right\} .
$$


The case where $\varphi_{j}$ has the form $(\exists x) \psi(x, \vec{y})$ is dealt with similarly. We then let

$$
\mathcal{P}^{\prime}=\bigcup_{j<\omega} \mathcal{P}_{j} \quad \text { and } \quad T^{\prime}=\bigcup_{j<\omega} T_{j} .
$$

Since $\models_{\mathbb{K}}$ is finitary, $T^{\prime} \nvdash_{\mathbb{K}} \Psi$. Moreover, for any formula of the form $(\forall x) \psi(x, \vec{y})$ or $(\exists x) \psi(x, \vec{y})$, we have, respectively,

$$
\bigwedge_{i=1}^{n} \psi\left(f_{i}^{\psi}(\vec{y}), \vec{y}\right) \leq(\forall x) \psi(x, \vec{y}) \in T^{\prime} \quad \text { or } \quad(\exists x) \psi(x, \vec{y}) \leq \bigvee_{i=1}^{n} \psi\left(f_{i}^{\psi}(\vec{y}), \vec{y}\right) \in T^{\prime},
$$

and as the converse inequations always hold, the claim follows.

To complete the proof of the theorem, we let $\hat{T}_{0}=T$ and $\hat{\mathcal{P}}_{0}=\mathcal{P}$, and define increasing sequences of languages $\hat{\mathcal{P}}_{i}$ and $\left\langle\mathcal{L}, \hat{\mathcal{P}}_{i}\right\rangle$-theories $\hat{T}_{i+1}$ as in the claim. The theory $\hat{T}=\bigcup_{i \in \mathbb{N}} \hat{T}_{i}$ then clearly has $n$-witnessed models and satisfies $\hat{T} \forall_{\mathbb{K}} \Psi$.

Note that only one-step Skolemization of degree $n$ for inequations of a certain form is needed for the proof of the converse direction.

Example 9. Recall that $\models_{[0,1]_{\mathrm{G}}}$ is finitary, so if it does not admit parallel Skolemization of degree 1, it does not have the 1 -witnessed model property or indeed, since $[0,1]_{\mathrm{G}}$ is a chain, the $n$-witnessed model property for any $n \in \mathbb{N}^{+}$. To show this, just note that (recalling that $\neg x=1$ if $x=0$ and 0 otherwise),

$$
\{\overline{1} \leq \neg \neg P(x), P(c) \leq \overline{0}\} \models_{[0,1]_{\mathrm{G}}} \overline{1} \leq \overline{0},
$$

but, considering a model with domain $\mathbb{N}$ where $P(i)=\frac{1}{i+1}$,

$$
\{\overline{1} \leq \neg \neg P(x),(\forall x) P(x) \leq \overline{0}\} \not_{[0,1]_{\mathrm{G}}} \overline{1} \leq \overline{0} .
$$

Finally, let us comment briefly on a generalization of these results. Rather than insisting that each consequence that fails, fails in an $n$-witnessed model for a fixed $n$, we might allow that it fails only in some finitely witnessed model. We say that the consequence relation $\models_{\mathbb{K}}$ has the $\omega$-witnessed model property if for any $\langle\mathcal{L}, \mathcal{P}\rangle$-theory $T \cup\{\Phi\}$,

$$
T \models_{\mathbb{K}} \Phi \Longleftrightarrow \text { every } n \text {-witnessed model } \mathfrak{M} \text { of } T \text { for any } n \in \mathbb{N} \text { is a model of } \Phi \text {. }
$$

Similarly, we can say that $\models_{\mathbb{K}}$ admits parallel Skolemization of degree $\omega$ if for any $\langle\mathcal{L}, \mathcal{P}\rangle$-theory $T \cup\{\Phi\}$ and any $\langle\mathcal{L}, \mathcal{P}\rangle$-inequation $\Psi$, there exists $n \in \mathbb{N}$ such that

$$
T \cup\{\Phi\} \vDash_{\mathbb{K}} \Psi \Longleftrightarrow T \cup\left\{\mathrm{sk}_{n}^{\forall}(\Phi)\right\} \vDash_{\mathbb{K}} \Psi .
$$

It is then relatively straightforward, adapting the above proofs, to prove that if $\models_{\mathbb{K}}$ has the $\omega$-witnessed model property, then it admits parallel Skolemization of degree $\omega$, and that the converse holds if $\models_{\mathbb{K}}$ is finitary. Note, however, that there exists no general method for determining a suitable $n$ for applying parallel Skolemization of degree $\omega$ to a given consequence, so this generalization does not seem to be of any practical value.

\subsection{Prewitnessed Model Properties}

In the previous subsection, we proved that consequence relations satisfying a rather strong witnessed model property admit parallel Skolemization to some degree for all formulas. In this section, we investigate the (broader) scope of parallel Skolemization restricted to prenex inequations, i.e., inequations between two prenex formulas.

First we show that a certain restricted form of parallel Skolemization for prenex inequations on the right holds in the presence of a weaker witnessed model property. Let $\boldsymbol{L}$ be a lattice and consider $\mathcal{X} \subseteq \mathcal{P}(L)$. We say that $\mathcal{X}$ is $n$-precompact for some $n \in \mathbb{N}^{+}$if for all $A \in \mathcal{X}$ and $b \in L$,

$$
\begin{aligned}
& \wedge A<b \quad \Longrightarrow \quad a_{1} \wedge \ldots \wedge a_{n}<b \quad \text { for some } a_{1}, \ldots, a_{n} \in A \\
& \vee A>b \quad \Longrightarrow \quad a_{1} \vee \ldots \vee a_{n}>b \quad \text { for some } a_{1}, \ldots, a_{n} \in A
\end{aligned}
$$




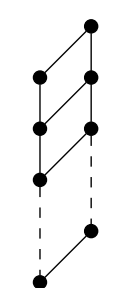

(a)

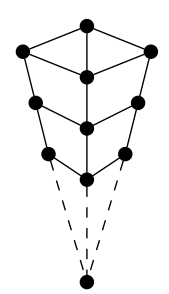

(b)

Figure 3: Examples of 1-precompact and 2-precompact systems

Example 10. The powerset of the (infinite) lattice depicted in Figure 3 a) is 1-precompact (but not $n$-compact for any $n$ ), while the powerset of the (infinite) lattice in Figure 3 b) is 2-precompact (but neither $n$-compact for any $n$, nor 1-precompact).

We call a $\mathcal{P}_{\text {-structure }} \subseteq=\langle\boldsymbol{A}, \mathbf{S}\rangle$-prewitnessed if the following set is $n$-precompact:

$$
\left\{\left\{\|\varphi(b, \bar{a})\|^{\mathscr{E}} \mid b \in S\right\} \mid \varphi(x, \bar{y}) \text { an }\langle\mathcal{L}, \mathcal{P}\rangle \text {-formula and } \vec{a} \subseteq S\right\} .
$$

Then $\models_{\mathbb{K}}$ has the $n$-prewitnessed model property if for any $\langle\mathcal{L}, \mathcal{P}\rangle$-theory $T \cup\{\Phi\}$,

$$
T \vDash_{\mathbb{K}} \Phi \Longleftrightarrow \text { every } n \text {-prewitnessed model } \mathfrak{M} \text { of } T \text { is a model of } \Phi \text {. }
$$

Example 11. If $\boldsymbol{L}$ is a chain, then $\mathcal{P}(L)$ is 1-precompact and hence any logic based on chains enjoys the 1-prewitnessed model property.

We show first that the $n$-prewitnessed model property suffices to guarantee "one step" parallel Skolemization of degree $n$ for inequations of a certain form occurring on the right of the consequence relation.

Lemma 9. Suppose that $\models_{\mathbb{K}}$ has the n-prewitnessed model property. Then for any $\langle\mathcal{L}, \mathcal{P}\rangle$-theory $T,\langle\mathcal{L}, \mathcal{P}\rangle$-formula $\varphi(x, \bar{y}),\langle\mathcal{L}, \mathcal{P}\rangle$-sentence $\psi$, and function symbols $f_{1}, \ldots, f_{n} \notin \mathcal{P}$ of arity $|\bar{y}|$

$$
\begin{gathered}
T \vDash_{\mathbb{K}} \psi \leq(\exists \bar{y})(\forall x) \varphi(x, \bar{y}) \Longleftrightarrow T \models_{\mathbb{K}} \psi \leq(\exists \bar{y})\left(\bigwedge_{i=1}^{n} \varphi\left(f_{i}(\bar{y}), \bar{y}\right)\right) \\
T \models_{\mathbb{K}}(\forall \bar{y})(\exists x) \varphi(x, \bar{y}) \leq \psi \Longleftrightarrow T \models_{\mathbb{K}}(\forall \bar{y})\left(\bigvee_{i=1}^{n} \varphi\left(f_{i}(\bar{y}), \bar{y}\right)\right) \leq \psi .
\end{gathered}
$$

Proof. We prove the first claim only; the second is dual. The left-to-right direction follows directly using Lemma 2 and we prove the right-to-left direction contrapositively.

Suppose that $T \forall_{\mathbb{K}} \psi \leq(\exists \bar{y})(\forall x) \varphi(x, \bar{y})$. Then there is an $n$-prewitnessed model $\mathfrak{M}=\langle\boldsymbol{A}, \mathbf{M}\rangle$ of $T$ such that $a=\|(\exists \bar{y})(\forall x) \varphi(x, \bar{y})\|^{\mathfrak{M}} ¥\|\psi\|^{\mathfrak{M}}=b$, i.e., $a<a \vee b$.

Suppose first that $a<a^{\prime}<a \vee b$ for some $a^{\prime} \in A$. Clearly, for each $\bar{m} \in M,\|(\forall x) \varphi(x, \bar{m})\|^{\mathfrak{M}} \leq a<a^{\prime}$. Since $\mathfrak{M}$ is $n$-prewitnessed, for each $\bar{m} \in M$, there are $u_{1}^{\bar{m}}, \ldots, u_{n}^{\bar{m}} \in M$ such that $\left\|\varphi\left(u_{1}^{\bar{m}}, \bar{m}\right)\right\|^{\mathfrak{M}} \wedge \ldots \wedge\left\|\varphi\left(u_{n}^{\bar{m}}, \bar{m}\right)\right\|^{\mathfrak{M}} \leq a^{\prime}$. Now for $i \in\{1, \ldots, n\}$, define, using the axiom of choice, $f_{i}(\bar{m})=u_{i}^{\bar{m}}$. But then

$$
\left\|(\exists \bar{y})\left(\bigwedge_{i=1}^{n} \varphi\left(f_{i}(\bar{y}), \bar{y}\right)\right)\right\|^{\mathfrak{M}}=\bigvee_{\bar{m} \in M} \bigwedge_{i=1}^{n}\left\|\varphi\left(f_{i}(\bar{m}), \bar{m}\right)\right\|^{\Re} \leq a^{\prime}<a \vee b .
$$

So $\left\|(\exists \bar{y})\left(\bigwedge_{i=1}^{n} \varphi\left(f_{i}(\bar{y}), \bar{y}\right)\right)\right\|^{\mathfrak{M}} \ngtr b$.

Now suppose that no $a^{\prime} \in A$ satisfies $a<a^{\prime}<a \vee b$. Clearly, for each $\bar{m} \in M,\|(\forall x) \varphi(x, \bar{m})\|^{\mathfrak{M}} \leq a<a \vee b$. If $\|(\forall x) \varphi(x, \bar{m})\|^{\mathfrak{M}}<a$, then, as $\mathfrak{M}$ is $n$-prewitnessed, we have $u_{1}^{\bar{m}}, \ldots, u_{n}^{\bar{m}} \in M$ such that $\left\|\varphi\left(u_{1}^{\bar{m}}, \bar{m}\right)\right\|^{\mathfrak{M}} \wedge \ldots \wedge\left\|\varphi\left(u_{n}^{\bar{m}}, \bar{m}\right)\right\|^{\mathfrak{M}} \leq$ $a$. If $\|(\forall x) \varphi(x, \bar{m})\|^{\mathfrak{M}}=a$, then for some $u_{1}^{\bar{m}}, \ldots, u_{n}^{\bar{m}} \in M$,

$$
\|(\forall x) \varphi(x, \bar{m})\|^{\mathfrak{M}}=a \leq\left\|\varphi\left(u_{1}^{\bar{m}}, \bar{m}\right)\right\|^{\mathfrak{M}} \wedge \ldots \wedge\left\|\varphi\left(u_{n}^{\bar{m}}, \bar{m}\right)\right\|^{\mathfrak{M}}<a \vee b .
$$


Hence, by assumption, $a=\left\|\varphi\left(u_{1}^{\bar{m}}, \bar{m}\right)\right\|^{\mathfrak{M}} \wedge \ldots \wedge\left\|\varphi\left(u_{n}^{\bar{m}}, \bar{m}\right)\right\|^{\mathfrak{M}}$. In both cases, for each $i \in\{1, \ldots, n\}$, define, using the axiom of choice, $f_{i}(\bar{m})=u_{i}^{\bar{m}}$. But then

$$
\left\|(\exists \bar{y})\left(\bigwedge_{i=1}^{n} \varphi\left(f_{i}(\bar{y}), \bar{y}\right)\right)\right\|^{\mathfrak{M}}=\bigvee_{\bar{m}} \bigwedge_{i=1}^{n}\left\|\varphi\left(f_{i}(\bar{m}), \bar{m}\right)\right\|^{\mathfrak{M}} \leq a<a \vee b .
$$

So $\left\|(\exists \bar{y})\left(\bigwedge_{i=1}^{n} \varphi\left(f_{i}(\bar{y}), \bar{y}\right)\right)\right\|^{\mathfrak{M}} \ngtr b$.

In order to repeat this one step Skolemization process and obtain Skolemized formulas for any prenex inequation, we require an additional assumption that $\models_{\mathbb{K}}$ admits certain quantifier shifts. This is the case, for example, when all the algebras in $\mathbb{K}$ are both frames and co-frames (i.e., joins distribute over infinite meets and vice-versa).

Theorem 2. Suppose that $\models_{\mathbb{K}}$ has the n-prewitnessed model property and that

$$
\vDash_{\mathbb{K}}(\chi \wedge(\exists x) \varphi) \approx(\exists x)(\chi \wedge \varphi) \text { and } \vDash_{\mathbb{K}}(\chi \vee(\forall x) \varphi) \approx(\forall x)(\chi \vee \varphi)
$$

Then for any predicate language $\mathcal{P},\langle\mathcal{L}, \mathcal{P}\rangle$-theory $T$, and prenex $\langle\mathcal{L}, \mathcal{P}\rangle$-inequation $\Phi$,

$$
T \models_{\mathbb{K}} \Phi \Longleftrightarrow T \models_{\mathbb{K}} \operatorname{sk}_{n}^{\exists}(\Phi)
$$

Proof. Let $\Phi=\varphi \leq \psi$. The left-to-right direction follows trivially. The right-to-left direction is a consequence of the following two claims:

$$
\begin{aligned}
& T \vDash_{\mathbb{K}} \varphi \leq \operatorname{sk}_{n}^{\exists}(\psi) \Longrightarrow T \vDash_{\mathbb{K}} \varphi \leq \psi, \\
& T \vDash_{\mathbb{K}} \operatorname{sk}_{n}^{\vee}(\varphi) \leq \psi \Longrightarrow T \models_{\mathbb{K}} \varphi \leq \psi .
\end{aligned}
$$

We prove just the first claim since the second is analogous. Let us define $\wedge$-prenex $\langle\mathcal{L}, \mathcal{P}\rangle$-formulas as follows: every quantifier-free $\langle\mathcal{L}, \mathcal{P}\rangle$-formula is $\wedge$-prenex, and if $\varphi, \psi$ are $\wedge$-prenex, then so are $\varphi \wedge \psi,(\exists x) \varphi$, and $(\forall x) \varphi$ for any variable $x$.

Now consider an $\langle\mathcal{L}, \mathcal{P}\rangle$-theory $T$, an $\langle\mathcal{L}, \mathcal{P}\rangle$-sentence $\alpha$, and a $\wedge$-prenex $\langle\mathcal{L}, \mathcal{P}\rangle$-sentence $\chi[(\forall x) \varphi(x, \bar{y})]$ with a leftmost strong quantifier occurrence $(\forall x) \varphi(x, \bar{y})$. We may assume that $\bar{y}=\bar{y}_{1}, \ldots, \bar{y}_{m}$, the variables $\bar{y}_{i}$ 's are all mutually disjoint and different from $x$, and

$$
\chi=\left(\exists \bar{y}_{1}\right)\left(\chi_{1}\left(\bar{y}_{1}\right) \wedge\left(\exists \bar{y}_{2}\right)\left(\chi_{2}\left(\bar{y}_{1}, \bar{y}_{2}\right) \wedge \ldots \wedge\left(\exists \bar{y}_{m}\right)\left(\chi_{m}(\bar{y}) \wedge(\forall x) \varphi(x, \bar{y})\right) \ldots\right) .\right.
$$

Then, using the assumed quantifier shifts, $\chi$ is equivalent to

$$
(\exists \bar{y})(\forall x)\left(\chi_{1}\left(\bar{y}_{1}\right) \wedge \chi_{2}\left(\bar{y}_{1}, \bar{y}_{2}\right) \wedge \ldots \wedge \chi_{m}(\bar{y}) \wedge \varphi(x, \bar{y})\right) .
$$

An application of Lemma 9 yields that $T \models_{\mathbb{K}} \alpha \leq \chi$ if and only if

$$
T \models_{\mathbb{K}} \alpha \leq(\exists \bar{y})\left(\chi_{1}\left(\bar{y}_{1}\right) \wedge \chi_{2}\left(\bar{y}_{1}, \bar{y}_{2}\right) \wedge \ldots \wedge \chi_{m}(\bar{y}) \wedge \bigwedge_{i=1}^{n} \varphi\left(f_{i}(\bar{y}), \bar{y}\right)\right) .
$$

But then, shifting the existential quantifiers back to their original positions,

$$
T \vDash_{\mathbb{K}} \alpha \leq \chi[(\forall x) \varphi(x, \bar{y})] \Longleftrightarrow T \vDash_{\mathbb{K}} \alpha \leq \chi\left[\bigwedge_{i=1}^{n} \varphi\left(f_{i}(\bar{y}), \bar{y}\right)\right] .
$$

Note that $\chi\left[\bigwedge_{i=1}^{n} \varphi\left(f_{i}(\bar{y}), \bar{y}\right)\right]$ is also a $\wedge$-prenex formula. Hence, the claim follows by an induction on the multiset of depths of quantifier occurrences according to the Derschowitz-Manna ordering (see Footnote6).

It is easy to see that if $\mathbb{K}$ is any class of chains, then $\models_{\mathbb{K}}$ has the 1-prewitnessed model property (as powersets of chains are 1-precompact) and proves the necessary quantifiers shifts, so we have: 
Corollary 2. Let $\mathbb{K}$ be a class of chains. Then for any predicate language $\mathcal{P},\langle\mathcal{L}, \mathcal{P}\rangle$-theory $T$, and prenex $\langle\mathcal{L}, \mathcal{P}\rangle$ inequation $\Phi$,

$$
T \vDash_{\mathbb{K}} \Phi \Longleftrightarrow T \models_{\mathbb{K}} \operatorname{sk}_{1}^{\exists}(\Phi) .
$$

On the other hand, powersets of chains need not be 1-compact, so we do not automatically get full parallel Skolemization of degree 1 ; indeed, we have already seen that $\mathbb{K}=\left\{[0,1]_{\mathrm{G}}\right\}$ is a class of chains for which $\models_{\mathbb{K}}$ does not admit full parallel Skolemization of any degree.

\section{Herbrand Theorems}

In the previous section, we showed how (parallel) Skolemization methods can be used to reduce consequences in first-order lattice-valued logics admitting certain witnessing properties to consequences without strong occurrences of quantifiers. In this section, we develop Herbrand theorems for performing a further reduction to consequences between propositional inequations. We first prove a general expansion lemma that performs this reduction on the left of consequences by introducing (typically infinitely many) propositional inequations. It is then proved that finitarity for propositional consequence corresponds to removing weak occurrences of quantifiers while introducing only finitely many propositional inequations. Our main Herbrand theorem then establishes that this reduction can be performed by taking finite conjunctions or disjunctions of instances of formulas, analogously to the classical case. Since consequence cannot be reduced in general to satisfiability in lattice-valued logics, we provide also a Herbrand theorem for satisfiability.

\subsection{A Herbrand Theorem for Consequence}

Let us fix again a lattice-oriented signature $\mathcal{L}$ and a predicate language $\mathcal{P}=\langle\mathbf{P}, \mathbf{F}, \mathbf{a r}\rangle$. We define the Herbrand universe $\mathcal{U}(\mathcal{P})$ to be the set of closed $\mathcal{P}$-terms, recalling that, by assumption, every predicate language contains at least one object constant and hence $\mathcal{U}(\mathcal{P}) \neq \emptyset$.

Let us assume now and for the rest of this section that $\mathbb{K}$ is a fixed class of complete $\mathcal{L}$-lattices. Recall from Lemma 5 that for any g-universal $\langle\mathcal{L}, \mathcal{P}\rangle$-inequation $\Phi$, there is a finite set $F(\Phi)$ of quantifier-free inequations such that for any theory $T \cup\{\Psi\}$,

$$
T \cup\{\Phi\} \models_{\mathbb{K}} \Psi \Longleftrightarrow T \cup F(\Phi) \vDash_{\mathbb{K}} \Psi .
$$

We show that given any set of g-universal $\langle\mathcal{L}, \mathcal{P}\rangle$-inequations on the left and a g-existential sentential $\langle\mathcal{L}, \mathcal{P}\rangle$-inequation on the right of the consequence relation, we can expand arbitrarily many of the inequations on the left by replacing variables with members of the Herbrand universe. In particular, any quantifier-free inequation on the left can be replaced by a (typically infinite) set of closed quantifier-free inequations.

Lemma 10 (Expansion Lemma). For each g-universal $\langle\mathcal{L}, \mathcal{P}\rangle$-theory $T \cup R$ and g-existential sentential $\langle\mathcal{L}, \mathcal{P}\rangle$ inequation $\Psi$,

$$
T \cup R \models_{\mathbb{K}} \Psi \Longleftrightarrow T \cup\{\Phi(\vec{t}, \vec{y}) \mid \Phi(\vec{x}, \vec{y}) \in R, \vec{t} \in \mathcal{U}(\mathcal{P})\} \models_{\mathbb{K}} \Psi .
$$

Proof. The right-to-left direction is straightforward since $\{\Phi(\vec{x}, \vec{y})\} \vDash_{\mathbb{K}} \Phi(\vec{t}, \vec{y})$ for each $\vec{t} \in \mathcal{U}(\mathcal{P})$. We prove the converse direction contrapositively. Suppose that there is a model $\mathfrak{M}=\langle\boldsymbol{A}, \mathbf{M}\rangle$ of

$$
S=T \cup\{\Phi(\vec{t}, \vec{y}) \mid \Phi(\vec{x}, \vec{y}) \in R, \vec{t} \in \mathcal{U}(\mathcal{P})\}
$$

such that $\mathfrak{M} \not \models \Psi$. Consider the substructure $\mathfrak{M}^{\prime}=\left\langle\boldsymbol{A}, \mathbf{M}^{\prime}\right\rangle$ of $\mathfrak{M}$ with domain $M^{\prime}=\left\{\|t\|^{\mathfrak{M}} \mid t \in \mathcal{U}(\mathscr{P})\right\}$. Then by Proposition $2 \mathfrak{M}^{\prime}$ is a model of $S$ such that $\mathfrak{M}^{\prime} \forall \Psi$. Consider $\Phi(\vec{x}, \vec{y}) \in R$. For each $\vec{b} \in M^{\prime}$ there is $\vec{t} \in \mathcal{U}(\mathcal{P})$ such that $\vec{b}=\|\vec{t}\|^{\mathfrak{M}}$ and $\mathfrak{M}^{\prime} \vDash \Phi(\vec{t}, \vec{y})$. So $\mathfrak{M}^{\prime} \vDash \Phi(\vec{x}, \vec{y})$. Hence $T \cup R \forall_{\mathbb{K}} \Psi$.

In combination with Skolemization, the Expansion Lemma can be used to show that a procedure for deciding consequences of finite sets of premises in propositional languages can be extended to inequations between one-variable first-order formulas without non-constant function symbols. Examples of classes $\mathbb{K}$ satisfying the premises of the following theorem include the classes of all finite MV-chains or G-chains or the class containing just the standard MV-algebra $[0,1]_{£}$ (Skolemization is obtained as a result of Theorem 1 and Examples 7 and 8, for the decision procedures see, e.g., [20]). 
Theorem 3. Suppose that $\models_{\mathbb{K}}$ is known to admit parallel Skolemization of degree $n$ for some $n \in \mathbb{N}$ and there is a decision procedure for finitary consequence on propositional inequations in $\models_{\mathbb{K}}$. Then finitary consequence on inequations between one-variable formulas without non-constant function symbols is decidable for $\models_{\mathbb{K}}$.

Proof. Suppose that we want to check $T \models_{\mathbb{K}} \varphi \leq \psi$ where $T \cup\{\varphi \leq \psi\}$ is a finite set of inequations between onevariable formulas without non-constant function symbols. Then any subformula of a formula in $T \cup\{\varphi \leq \psi\}$ of the form $(\forall x) \chi$ or $(\exists x) \chi$ contains no free variables, and Skolemization gives (constructively) a finite set of g-universal inequations $T^{\prime}$ and a g-existential inequation $\left\{\varphi^{\prime} \leq \psi^{\prime}\right\}$ containing no non-constant function symbols such that

$$
T \models_{\mathbb{K}} \varphi \leq \psi \Longleftrightarrow T^{\prime} \vDash_{\mathbb{K}} \varphi^{\prime} \leq \psi^{\prime} .
$$

But then the Herbrand universe of the new language is finite and by the Expansion Lemma, we obtain a finite set of ground inequations $T^{\prime \prime} \cup\left\{\varphi^{\prime \prime} \leq \psi^{\prime \prime}\right\}$ such that

$$
T^{\prime} \vDash \models_{\mathbb{K}} \varphi^{\prime} \leq \psi^{\prime} \Longleftrightarrow T^{\prime \prime} \vDash_{\mathbb{K}} \varphi^{\prime \prime} \leq \psi^{\prime \prime}
$$

By assumption, there is a decision procedure for finitary consequence on inequations between propositional formulas in $\models_{\mathbb{K}}$, so also finitary consequence on one-variable formulas without non-constant function symbols is decidable for $\vDash \mathbb{K}$.

More generally, Lemma 10 may be viewed as a Herbrand theorem for lattice-valued logics that removes free variables (or weak quantifiers) by introducing (typically infinitely many) formulas where the free variables are replaced by closed terms. A more familiar version of the Herbrand theorem then amounts to the claim that a finite subset of these formulas suffices, that is, to the claim that a certain form of finitarity holds for the logic. In fact, it turns out that it is enough to require finitarity only with respect to propositional languages. To distinguish this property from the one we have seen in the previous section, let us say that $\mathbb{K}$ is finitary if for each propositional language $\mathcal{P}$ and $\mathcal{P}$-theory $T \cup\{\Psi\}$ :

$$
T \models_{\mathbb{K}}^{\mathcal{P}} \Psi \Longleftrightarrow T^{\prime} \vDash_{\mathbb{K}}^{\mathcal{P}} \Psi \text { for some finite } T^{\prime} \subseteq T .
$$

Remark 4. Of course $\mathbb{K}$ is finitary if $\models_{\mathbb{K}}$ is finitary, and we conjecture that the converse claim fails even though we are not aware of any particular example. Sufficient conditions for the finitarity of $\mathbb{K}$ are obtained by weakening those stated in Proposition 1 and Example 2 by requiring only the existence of any, not necessarily regular, embedding. However, in this case we can also give a full algebraic characterization. Clearly, $\mathbb{K}$ is finitary if and only if equational consequence for the class of algebras $\mathbb{K}$ is finitary. This latter condition is known to be equivalent to the claim that the generalized quasivariety generated by $\mathbb{K}$ (i.e., a class axiomatized by generalized quasiequations with countably many premises valid in $\mathbb{K}$ ) coincides with the quasivariety generated by $\mathbb{K}$, i.e., $\mathbf{I S P P}_{u}(\mathbb{K})=\mathbf{I S P}_{\sigma-f}(\mathbb{K})$, where $\mathbf{P}_{\sigma-f}(\mathbb{K})$ is the class of reduced products of $\mathbb{K}$ over a filter closed under countable intersections 7 The last equality can be replaced by simple subsethood $\mathbf{P}_{u}(\mathbb{K}) \subseteq \mathbf{I S P}_{\sigma-f}(\mathbb{K})$, in words: any ultraproduct of elements of $\mathbb{K}$ can be embedded into a $\sigma$-filtered product of elements of $\mathbb{K}$ (an obvious consequence of both 'non-regular' versions of the sufficient conditions from Proposition 1 .

We show first that this notion of finitarity extends to a more general first-order setting.

Lemma 11. If $\mathbb{K}$ is finitary, then for each g-universal $\langle\mathcal{L}, \mathcal{P}\rangle$-theory $T$ and g-existential sentential $\langle\mathcal{L}, \mathcal{P}\rangle$-inequation $\Psi$,

$$
T \vDash_{\mathbb{K}} \Psi \Longleftrightarrow T^{\prime} \vDash_{\mathbb{K}} \Psi \text { for some finite } T^{\prime} \subseteq T \text {. }
$$

Proof. Suppose that $\Psi=\varphi \leq \psi$. First we show that we can assume that $\varphi$ and $\psi$ are propositional atoms. Indeed, if $T \vDash_{\mathbb{K}} \varphi \leq \psi$, then by Corollary $1, T \cup\{P \leq \varphi, \psi \leq Q\} \vDash_{\mathbb{K}} P \leq Q$ for some new propositional atoms $P$ and $Q$. But then if $T^{\prime} \cup\{P \leq \varphi, \psi \leq Q\} \models_{\mathbb{K}} P \leq Q$ for some finite $T^{\prime} \subseteq T$, again by Corollary 1$] T^{\prime} \models_{\mathbb{K}} \varphi \leq \psi$.

\footnotetext{
${ }^{7}$ The restriction to countable notions comes from our standing assumption that predicate languages are countable and from assuming that the equational consequence is defined over a countable set of object variables.
} 
Suppose then that $T \models_{\mathbb{K}} P \leq Q$ for propositional atoms $P$ and $Q$. Using Lemma 5 for each $\Psi \in T$ we obtain a finite quantifier-free theory $F(\Psi)$ such that $F(\Psi) \models_{\mathbb{K}} \Psi$, and hence

$$
\bigcup_{\Psi \in T} F(\Psi) \vDash_{\mathbb{K}} P \leq Q .
$$

Using Lemma 10, we obtain

$$
\bigcup_{\Psi \in T}\left\{\chi(\vec{t}) \leq \chi^{\prime}(\vec{t}) \mid \chi(\vec{x}) \leq \chi^{\prime}(\vec{x}) \in F(\Psi) \text { and } \vec{t} \in \mathcal{U}(\mathcal{P})\right\} \models_{\mathbb{K}} P \leq Q .
$$

All the formulas in this consequence are quantifier-free sentences. Hence, using Lemma 4 and the finitarity of $\mathbb{K}$, for some finite $T^{\prime} \subseteq T$,

$$
\bigcup_{\Psi \in T^{\prime}}\left\{\chi(\vec{t}) \leq \chi^{\prime}(\vec{t}) \mid \chi(\vec{x}) \leq \chi^{\prime}(\vec{x}) \in F(\Psi) \text { and } \vec{t} \in \mathcal{U}(\mathcal{P})\right\} \models_{\mathbb{K}} P \leq Q .
$$

But then by Lemma 10

$$
\bigcup_{\Psi \in T^{\prime}} F(\Psi) \models_{\mathbb{K}} P \leq Q
$$

and Lemma 5 completes the proof.

We are able now to prove the mentioned equivalence between finitarity and a general Herbrand theorem for latticevalued logics.

Proposition 3. The following are equivalent:

(1) $\mathbb{K}$ is finitary.

(2) For each g-universal $\langle\mathcal{L}, \mathcal{P}\rangle$-theory $T \cup\{\Phi(\vec{x})\}$ and g-existential sentential $\langle\mathcal{L}, \mathcal{P}\rangle$-inequation $\Psi$,

$$
T \cup\{\Phi(\vec{x})\} \models_{\mathbb{K}} \Psi \Longleftrightarrow T \cup\{\Phi(\vec{t}) \mid \vec{t} \in H\} \vDash_{\mathbb{K}} \Psi \text { for some finite } H \subseteq \mathcal{U}(\mathcal{P}) \text {. }
$$

Proof. $(1) \Rightarrow$ (2) follows directly from Lemmas 10 and 11. To prove (2) $\Rightarrow$ (1), let us fix a propositional language $\mathcal{P}_{0}$ and define a predicate language $\mathcal{P}$ with a unary predicate symbol $P$ such that $\mathcal{U}(\mathcal{P})$ is countably infinite. Without loss of generality we can assume that $\mathcal{P}_{0}$ is also infinite. We list the elements of $\mathcal{U}(\mathcal{P})$ as $t_{i}$ and the elements of $\mathcal{P}_{0}$ as $P_{i}$ with $i \in \mathbb{N}$. Let $\left\{\varphi_{i} \leq \psi_{i} \mid i \in \mathbb{N}\right\} \cup\{\Psi\}$ be a set of propositional inequations such that

$$
\left\{\varphi_{i} \preceq \psi_{i} \mid i \in \mathbb{N}\right\} \models_{\mathbb{K}}^{\mathcal{P}_{0}} \Psi
$$

Since $\left\{P(x) \leq Q(x), \varphi_{i} \leq P\left(t_{i}\right), Q\left(t_{i}\right) \leq \psi_{i}\right\} \models_{\mathbb{K}} \varphi_{i} \leq \psi_{i}$, we obtain

$$
\{P(x) \leq Q(x)\} \cup\left\{\varphi_{i} \leq P\left(t_{i}\right), Q\left(t_{i}\right) \leq \psi_{i} \mid i \in \mathbb{N}\right\} \models_{\mathbb{K}} \Psi .
$$

Then by assumption, for some $n \in \mathbb{N}$,

$$
\left\{P\left(t_{i}\right) \leq Q\left(t_{i}\right) \mid i \leq n\right\} \cup\left\{\varphi_{i} \leq P\left(t_{i}\right), Q\left(t_{i}\right) \leq \psi_{i} \mid i \in \mathbb{N}\right\} \models_{\mathbb{K}} \Psi .
$$

We define a $\mathcal{P}_{0} \cup \mathcal{P}$-substitution $\sigma$ that satisfies $\sigma\left(P_{3 k}\right)=P\left(t_{k}\right), \sigma\left(P_{3 k+1}\right)=Q\left(t_{k}\right), \sigma\left(P_{3 k+2}\right)=P_{k}$, and is the identity otherwise, and, using the second part of Lemma 4, obtain

$$
\left\{P_{3 i} \leq P_{3 i+1} \mid i \leq n\right\} \cup\left\{\bar{\varphi}_{i} \leq P_{3 i}, P_{3 i+1} \leq \bar{\psi}_{i} \mid i \in \mathbb{N}\right\} \vDash \mathbb{K} \Psi
$$

where $\bar{\varphi}_{i}$ and $\bar{\psi}_{i}$ are the formulas resulting from $\varphi_{i}$ and $\psi_{i}$, respectively, by replacing propositional atoms $P_{k}$ by $P_{3 k+2}$. Finally, we use the first part of Lemma 4 and the substitution $\sigma\left(P_{3 k}\right)=\varphi_{k}, \sigma\left(P_{3 k+1}\right)=\psi_{k}$, and $\sigma\left(P_{3 k+2}\right)=P_{k}$ to obtain

$$
\left\{\varphi_{i} \leq \psi_{i} \mid i \leq n\right\} \models_{\mathbb{K}} \Psi .
$$


Herbrand theorems are often formulated as a reduction from a formula with free variables or weak quantifiers to a conjunction or disjunction of ground instances of the formula. Such theorems can also be obtained in the context of lattice-valued logics. Let the $\langle\mathcal{L}, \mathcal{P}\rangle$-Herbrand expansion $E(\varphi)$ of an $\langle\mathcal{L}, \mathcal{P}\rangle$-formula $\varphi$ consist of all formulas obtained by applying the following two steps repeatedly, starting with $\varphi$, until no quantifiers remain:

(I) Replace $\psi[(\forall \vec{x}) \chi(\vec{x}, \vec{y})]$ where $\chi$ is quantifier-free with $\psi\left[\bigwedge_{\vec{t} \in H} \chi(\vec{t}, \vec{y})\right]$ for some finite $H \subseteq \mathcal{U}(\mathcal{P})$.

(II) Replace $\psi[(\exists \vec{x}) \chi(\vec{x}, \vec{y})]$ where $\chi$ is quantifier-free with $\psi\left[\bigvee_{\vec{t} \in H} \chi(\vec{t}, \vec{y})\right]$ for some finite $H \subseteq \mathcal{U}(\mathcal{P})$.

Notice that if $\varphi$ is a sentence, then so are all formulas in $E(\varphi)$. We extend the definition of an $\langle\mathcal{L}, \mathcal{P}\rangle$-Herbrand expansion to inequations by

$$
E(\varphi(\vec{x}) \leq \psi(\vec{x}))=\left\{\left\{\varphi^{\prime}(\vec{t}) \leq \psi^{\prime}(\vec{t}) \mid \vec{t} \in H\right\} \mid \varphi^{\prime}, \psi^{\prime} \in E(\psi), \text { finite } H \subseteq \mathcal{U}(\mathcal{P})\right\} .
$$

If $\varphi$ and $\psi$ are sentences, then $E(\varphi \leq \psi)=\left\{\varphi^{\prime} \leq \psi^{\prime} \mid \varphi^{\prime} \in E(\varphi)\right.$ and $\left.\psi^{\prime} \in E(\psi)\right\}$.

A simple induction, using Lemma 2 and the fact that $\models_{\mathbb{K}}(\forall \vec{x}) \chi(\vec{x}, \vec{y}) \leq \bigwedge_{\vec{t} \in H} \chi(\vec{t}, \vec{y})$ and $\models_{\mathbb{K}} \bigvee_{\vec{t} \in H} \chi(\vec{t}, \vec{y}) \leq$ $(\exists \vec{x}) \chi(\vec{x}, \vec{y})$, provides the following analogue of Lemma 6 .

Lemma 12. For any $\langle\mathcal{L}, \mathcal{P}\rangle$-formula $\varphi$ and $\varphi^{\prime} \in E(\varphi)$, and any g-universal $\langle\mathcal{L}, \mathcal{P}\rangle$-inequation $\Phi$ and $\Phi^{\prime} \in E(\Phi)$,

(i) $\models_{\mathbb{K}} \varphi \leq \varphi^{\prime}$ if $\varphi$ is g-universal, and $\models_{\mathbb{K}} \varphi^{\prime} \leq \varphi$ if $\varphi$ is g-existential;

(ii) $\Phi \models_{\mathbb{K}} \Psi$ for each $\Psi \in \Phi^{\prime}$.

We are now able to establish our main Herbrand theorem for premises and conclusions of consequences in latticevalued logics.

Theorem 4 (Herbrand Theorem for Consequence). Let $\mathbb{K}$ be a finitary class of complete $\mathcal{L}$-lattices. Then for every g-universal $\langle\mathcal{L}, \mathcal{P}\rangle$-theory $T \cup\{\Phi\}$ and g-existential sentential $\langle\mathcal{L}, \mathcal{P}\rangle$-inequation $\Psi$,

$$
\begin{aligned}
T \cup\{\Phi\} \vDash_{\mathbb{K}} \Psi & \Longleftrightarrow T \cup\left\{\Phi^{\prime}\right\} \vDash_{\mathbb{K}} \Psi \text { for some } \Phi^{\prime} \in E(\Phi) \\
T \models_{\mathbb{K}} \Psi & \Longleftrightarrow T \models_{\mathbb{K}} \Psi^{\prime} \text { for some } \Psi^{\prime} \in E(\Psi),
\end{aligned}
$$

and we say that $\models_{\mathbb{K}}$ admits the Herbrand theorem for consequence.

Proof. The right-to-left direction in both cases follows directly using Lemma 12 Moreover, the left-to-right direction of the second case follows easily from the first case. Suppose that $\Psi=\varphi \leq \psi$. Since $\varphi \leq \psi$ is sentential, by Corollary 1 there are new propositional atoms $P$ and $Q$ such that $T \cup\{P \leq \varphi\} \cup\{\psi \leq Q\} \vDash_{\mathbb{K}} P \leq Q$. Using the first case, we obtain $\varphi^{\prime} \in E(\varphi)$ and $\psi^{\prime} \in E(\psi)$ such that $T \cup\left\{P \leq \varphi^{\prime}\right\} \cup\left\{\psi^{\prime} \leq Q\right\} \vDash_{\mathbb{K}} P \leq Q$, and a further application of Corollary 1 completes the proof.

It remains then to prove the left-to-right direction of the first case. Suppose that $\Phi=\varphi \leq \psi$. Using Lemma 5 , we obtain a finite set of quantifier-free inequations $F(\varphi \leq \psi)$ and can then apply Proposition 3 to obtain a finite set of inequations with free variables replaced by elements of the Herbrand universe. However, to see that we obtain exactly the formulas we need, we must consider again the induction step of the proof of Lemma 5 .

Recall that we proceed by induction on the number of quantifiers in $\varphi \leq \psi$. For the induction step, we suppose that $\varphi$ has a proper universal subformula $(\forall \vec{x}) \chi(\vec{x}, \vec{y})$, i.e., $\varphi=\varphi[(\forall \vec{x}) \chi(\vec{x}, \vec{y})]^{-}$(the proof for other cases is analogous). A new predicate symbol $P_{\chi}$ of the appropriate arity (the length of $\vec{y}$ ) is introduced such that

$$
T \cup\left\{P_{\chi}(\vec{y}) \leq \chi(\vec{x}, \vec{y}), \varphi\left[P_{\chi}(\vec{y})\right] \leq \psi\right\} \vDash_{\mathbb{K}} \Psi .
$$

Using Proposition 3 for $T \cup\left\{\varphi\left[P_{\chi}(\vec{y})\right] \leq \psi\right\}$ and $P_{\chi}(\vec{y}) \leq \chi(\vec{x}, \vec{y})$, we obtain now a finite $H \subseteq \mathcal{U}(\mathcal{P})$ such that

$$
T \cup\left\{\varphi\left[P_{\chi}(\vec{y})\right] \leq \psi\right\} \cup\left\{P_{\chi}(\vec{y}) \leq \chi(\vec{t}, \vec{y}) \mid \vec{t} \in H\right\} \models_{\mathbb{K}} \Psi .
$$

So also using the properties of $\wedge$ we obtain

$$
T \cup\left\{\varphi[(\forall \vec{x}) \chi(\vec{x}, \vec{y})] \leq \psi, P_{\chi}(\vec{y}) \leq \bigwedge_{\vec{t} \in H} \chi(\vec{t}, \vec{y})\right\} \models_{\mathbb{K}} \Psi .
$$


By Lemma 4 using a substitution $\sigma$ that satisfies $\sigma\left(P_{\chi}\right)=\bigwedge_{\vec{t} \in H} \chi(\vec{t}, \vec{y})$ and is the identity otherwise, it follows that

$$
T \cup\left\{\sigma\left(\varphi\left[P_{\chi}(\vec{y})\right] \leq \psi\right)\right\} \models_{\mathbb{K}} \Psi .
$$

To complete the proof, note that the induction hypothesis can be applied to $\sigma\left(\varphi\left[P_{\chi}(\vec{y})\right]\right)=\varphi\left[\bigwedge_{\vec{t} \in H} \chi(\vec{t}, \vec{y})\right]$. By repeating this process until we obtain a quantifier-free inequation, we obtain appropriate elements of $E(\varphi)$ and $E(\psi)$. A further application of Proposition 3 yields the desired result.

Note that if the Herbrand theorem for consequence holds for $\models_{\mathbb{K}}$, then condition (2) of Proposition 3 is satisfied, so $\mathbb{K}$ is finitary.

We conclude this subsection by establishing the failure of the Herbrand theorem for consequence, and therefore also of finitarity, for a wide range of substructural logics. More precisely, we show that these properties cannot hold for any lattice-valued logic defined by a class of $\mathrm{FL}_{\mathrm{e}}$-chains (see Example 1 ) that admits certain quantifier shifts and contains arbitrarily large members (for example, logics based on classes of finite $\mathrm{FL}_{\mathrm{e}}$-algebras containing chains of increasing size).

Theorem 5. Suppose that $\mathbb{K}$ is a class of $\mathrm{FL}_{\mathrm{e}}$-chains satisfying

(i) $\{\overline{1} \leq(\forall x) \varphi \rightarrow \psi\} \models_{\mathbb{K}} \overline{1} \leq(\exists x)(\varphi \rightarrow \psi)$ where $x$ is not free in $\psi$;

(ii) for each $n \in \mathbb{N}$, there exists $\boldsymbol{A} \in \mathbb{K}$ such that $|A| \geq n$.

Then $\mathbb{K}$ does not admit the Herbrand theorem for consequence and is not finitary.

Proof. Consider a first-order language with a unary predicate symbol $P$ and a constant symbol $c$. Since $\models_{\mathbb{K}} \overline{1} \leq$ $(\forall x) P(x) \rightarrow(\forall y) P(y)$, using (i) and Lemma 1 also $\models_{\mathbb{K}} \overline{1} \leq(\exists x)(\forall y)(P(x) \rightarrow P(y))$. Because $\mathbb{K}$ is a class of FL $\mathrm{e}^{-}$ chains, we can apply Corollary 2 and obtain $\models_{\mathbb{K}} \overline{1} \leq(\exists x)(P(x) \rightarrow P(f(x)))$.

Suppose now for a contradiction that $\mathbb{K}$ does admit the Herbrand theorem for consequence, noting that this is the case if and only if $\mathbb{K}$ is finitary. Then for some $n \in \mathbb{N}$, we obtain $\models_{\mathbb{K}} \overline{1} \leq \bigvee_{i \leq n}\left(P\left(f^{i}(c)\right) \rightarrow P\left(f^{i+1}(c)\right)\right)$. Let $\boldsymbol{A}$ be a member of $\mathbb{K}$ with a descending chain of elements $a_{1}, \ldots, a_{n+1}$. We define a model $\mathfrak{M}$ over $\boldsymbol{A}$ whose domain is the Herbrand universe such that $P^{\mathfrak{M}}\left(f^{i}(c)\right)=a_{i}$ for $i \leq n+1$. Then $\left\|P\left(f^{i}(c)\right) \rightarrow P\left(f^{i+1}(c)\right)\right\|^{\mathfrak{M}}=a_{i} \rightarrow a_{i+1}<\overline{1}^{A}$ and so $\mathfrak{M} \not \models \bigvee_{i \leq n}\left(P\left(f^{i}(c)\right) \rightarrow P\left(f^{i+1}(c)\right)\right)$, a contradiction.

Note that the assumption that $\mathbb{K}$ is a class of chains can be broadened to any class satisfying the assumptions of Theorem 2 ,

\subsection{A Herbrand Theorem for Satisfiability}

The standard Herbrand theorem for first-order classical logic may be stated equivalently in terms of satisfiability of sets of formulas rather than as a property of logical consequence. As remarked already in the introduction, this is not the case in general for lattice-valued logics. Indeed, as we will see below, there are cases where $\mathbb{K}$ is not finitary and the Herbrand theorem for consequence fails, but a Herbrand theorem for satisfiability nevertheless holds.

Let us assume (harmlessly) for this subsection that our language $\mathcal{L}$ contains constants $\perp$ and $\mathrm{T}$ interpreted in each $\mathcal{L}$-lattice as the bottom and top element. Furthermore we assume that $\mathbb{K}$ contains at least one non-trivial algebra, and hence $\forall_{\mathbb{K}} T \leq \perp$.

We say that a $\langle\mathcal{L}, \mathcal{P}\rangle$-theory $T$ is $\mathbb{K}$-satisfiable if it has a $\mathcal{P}$ - $\mathbb{K}$-model, or, equivalently, $T \forall_{\mathbb{K}} T \leq \perp$. Since $T \leq \perp$ is a g-existential sentential $\langle\mathcal{L}, \mathcal{P}\rangle$-inequation, the Expansion Lemma (Lemma 10p yields the following analogue for satisfiability.

Lemma 13 (Expansion Lemma for Satisfiability). For each g-universal $\langle\mathcal{L}, \mathcal{P}\rangle$-theory $T \cup R$, the following are equivalent:

(1) $T \cup\{\Phi(\vec{x}, \vec{y}) \mid \Phi(\vec{x}, \vec{y}) \in R\}$ is $\mathbb{K}$-satisfiable.

(2) $T \cup\{\Phi(\vec{t}, \vec{y}) \mid \Phi(\vec{x}, \vec{y}) \in R, \vec{t} \in \mathcal{U}(\mathcal{P})\}$ is $\mathbb{K}$-satisfiable. 
Let us say that $\mathbb{K}$ is compact if for each propositional language $\mathcal{P}$ and $\mathcal{P}$-theory $T$,

$T$ is $\mathbb{K}$-satisfiable $\Longleftrightarrow$ every finite subset of $T$ is $\mathbb{K}$-satisfiable.

Mimicking the proofs of Lemma 3 and Theorem 4 with $\Psi=T \leq \perp$ and using compactness rather than finitarity, we obtain the following results.

Proposition 4. The following are equivalent:

(1) $\mathbb{K}$ is compact.

(2) A g-universal $\langle\mathcal{L}, \mathcal{P}\rangle$-theory $T \cup\{\Phi(\vec{x})\}$ is $\mathbb{K}$-satisfiable, if and only if, for each finite $H \subseteq \mathcal{U}(\mathcal{P})$ the theory $T \cup\{\Phi(\vec{t}) \mid \vec{t} \in H\}$ is $\mathbb{K}$-satisfiable.

Theorem 6. If $\mathbb{K}$ is compact, then for every g-universal $\langle\mathcal{L}, \mathcal{P}\rangle$-theory $T \cup\{\Phi\}$,

$$
T \cup\{\Phi\} \text { is } \mathbb{K} \text {-satisfiable } \Longleftrightarrow T \cup \Phi^{\prime} \text { is } \mathbb{K} \text {-satisfiable for every } \Phi^{\prime} \in E(\Phi) \text {, }
$$

and we say that $\models_{\mathbb{K}}$ admits the Herbrand theorem for satisfiability.

Clearly if $\mathbb{K}$ is finitary, then it is compact, but the converse direction does not hold. Consider, for example, the class consisting of just the standard algebra $[0,1]_{七}$ of Łukasiewicz logic (see Example 1 ). Compactness for this class is proved in [18, Theorem 5.4.24], while the failure of finitarity (a folklore result; see, e.g., [18, Remark 3.2.15]) follows from Theorem 5 Hence $\models_{[0,1]_{\llcorner}}$is an example of a logic enjoying the Herbrand theorem for satisfiability but not the Herbrand theorem for consequence. In the remainder of this subsection, we show that we can use the former theorem to obtain also a new proof of an "approximate" Herbrand theorem for consequence in $[0,1]_{\mathrm{E}}$, considered (in a slightly weaker form) in [4].

First we introduce a useful notion of approximate validity for $[0,1]_{\mathrm{E}}$, defining for $r \in[0,1] \cap \mathbb{Q}$,

$$
T \vDash_{\mathrm{E}} r<\psi: \Longleftrightarrow \text { for every }[0,1]_{\mathrm{E}} \text {-model } \mathfrak{M} \text { of } T, r<\|\psi\|^{\mathfrak{M}} \text {. }
$$

Lemma 14. For any $r \in[0,1] \cap \mathbb{Q}$, there exists a quantifier-free formula $\chi_{r}^{P}$ containing just one propositional atom $P$ such that for any theory $T \cup\{\psi\}$ in which $P$ does not occur,

$$
T \vDash_{\mathrm{E}} r<\psi \Longleftrightarrow T \cup\left\{\psi \leq \chi_{r}^{P}\right\} \text { is }[0,1]_{\mathrm{E}} \text {-unsatisfiable. }
$$

Proof. Fix $r \in[0,1] \cap \mathbb{Q}$. By McNaughton's theorem [22], there exists for each piecewise linear function $f:[0,1] \rightarrow$ $[0,1]$ with integer coefficients a one-variable formula $\chi_{f}$ of propositional Łukasiewicz logic such that $\chi^{[0,1]_{ \pm}}(a)=f(a)$ for each $a \in[0,1]$. In particular, for $r=\frac{m}{n}\left(m \in \mathbb{N}, n \in \mathbb{N}^{+}, m \leq n\right)$ we consider the function $r(x)=\min \{x,(1-n) x+m\}$ and obtain a formula $\chi_{r}$ such that $\chi_{r}(r)=r$ and $\chi_{r}(d) \leq r$ for all $d \in[0,1]$. Now let $\chi_{r}^{P}$ be the result of replacing $p$ with a nullary predicate symbol $P$ in $\chi_{r}$.

To prove the claim, observe first that if $T \models_{\mathrm{E}} r<\psi$ and $\mathfrak{M}$ is a $[0,1]_{\mathrm{E}}$-model of $T$, then $r<\|\psi\|^{\mathfrak{M}}$ and $\left\|\chi_{r}^{P}\right\|^{\mathfrak{M}} \leq r$, so $\mathfrak{M}$ cannot be a model of $T \cup\left\{\psi \leq \chi_{r}^{P}\right\}$. For the converse direction, we proceed contrapositively. Suppose that $T \Downarrow_{\mathrm{E}} r<\psi$. Then there is a $[0,1]_{\mathrm{E}}$-model $\mathfrak{M}$ of $T$ such that $\|\psi\|^{\mathfrak{M}} \leq r$. Expand this model by setting $\|P\|^{\mathfrak{M}}=r$ and we obtain a $[0,1]_{\mathrm{E}}$-model of $\psi \leq \chi_{r}^{P}$. Hence $T \cup\left\{\psi \leq \chi_{r}^{P}\right\}$ is $[0,1]_{\mathrm{E}}$-satisfiable.

It follows that the approximate consequence relation for $r \in[0,1] \cap \mathbb{Q}$ can be defined in terms of satisfiabilty of sets of formulas in $\models_{[0,1]_{\mathrm{E}}}$. Moreover, the following proposition and approximate Herbrand theorem for Łukasiewicz logic are now immediate consequences of Lemma 14 and Theorem 6 .

Proposition 5. For each g-universal theory $T \cup\{\varphi\}$, g-existential formula $\psi$, and $r \in[0,1] \cap \mathbb{Q}$,

$$
\begin{aligned}
T \models_{\mathrm{E}} r<\psi & \Longleftrightarrow T \models_{\mathrm{E}} r<\psi^{\prime} \text { for some } \psi^{\prime} \in E(\psi), \\
T \cup\{\overline{1} \leq \varphi\} \models_{\mathrm{E}} r<\psi & \Longleftrightarrow T \cup\left\{\overline{1} \leq \varphi^{\prime}\right\} \models_{\mathrm{E}} r<\psi \text { for some } \varphi^{\prime} \in E(\varphi) .
\end{aligned}
$$

Theorem 7. For each g-universal theory $T \cup\{\varphi\}$ and g-existential formula $\psi$,

$$
\begin{aligned}
T \models_{[0,1]_{\mathrm{E}}} \overline{1} \leq \psi & \Longleftrightarrow \text { for each } n \in \mathbb{N} \text {, there exists } \psi^{\prime} \in E(\psi) \text { such that } T \models_{\mathrm{E}} \frac{n}{n+1}<\psi^{\prime}, \\
T \cup\{\overline{1} \leq \varphi\} \models_{[0,1]_{\mathrm{E}}} \overline{1} \leq \psi & \Longleftrightarrow \text { for each } n \in \mathbb{N} \text {, there exists } \varphi^{\prime} \in E(\varphi) \text { such that } T \cup\left\{\overline{1} \leq \varphi^{\prime}\right\} \models_{\mathrm{E}} \frac{n}{n+1}<\psi .
\end{aligned}
$$




\section{References}

[1] Baader, F., Brandt, S., Lutz, C., 2005. Pushing the $\mathcal{E} \mathcal{L}$ envelope, in: Kaelbling, L.P., Saffiotti, A. (Eds.), Proceedings of the 19th International Joint Conference on Artificial Intelligence (IJCAI 2005), Morgan Kaufmann, San Francisco, CA. pp. 364-369.

[2] Baaz, M., Iemhoff, R., 2008. On Skolemization in constructive theories. Journal of Symbolic Logic 73, $969-998$.

[3] Baaz, M., Iemhoff, R., 2016. Skolemization in intermediate logics with the finite model property. Logic Journal of the Interest Group of Pure and Applied Logic 3, 224-237.

[4] Baaz, M., Metcalfe, G., 2010. Herbrand's theorem, Skolemization, and proof systems for first-order Łukasiewicz logic. Journal of Logic and Computation 20, 35-54.

[5] Buss, S. (Ed.), 1998. Handbook of Proof Theory. Kluwer.

[6] Caicedo, X., . Lindström's theorems for Łukasiewicz predicate logic. To appear in Fundamenta Mathematicae.

[7] Ciabattoni, A., Galatos, N., Terui, K., 2012. Algebraic proof theory for substructural logics: Cut-elimination and completions. Annals of Pure and Applied Logic 163, 266-290.

[8] Cintula, P., Diaconecsu, D., Metcalfe, G., 2015. Skolemization for substructural logics, in: Davis, M., Fehnker, A., McIver, A., Voronkov, A. (Eds.), Proceedings of LPAR-20, Springer. pp. 1-15.

[9] Cintula, P., Metcalfe, G., 2013. Herbrand theorems for substructural logics, in: McMillan, K.L., Middeldorp, A., Voronkov, A. (Eds.), Proceedings of LPAR-19, Springer. pp. 584-600.

[10] Cintula, P., Noguera, C., 2011. A general framework for mathematical fuzzy logic, in: Cintula, P., Hájek, P., Noguera, C. (Eds.), Handbook of Mathematical Fuzzy Logic - Volume 1. College Publications, London. volume 37 of Studies in Logic, Mathematical Logic and Foundations, pp. 103-207.

[11] Cintula, P., Noguera, C., 2015. A Henkin-style proof of completeness for first-order algebraizable logics. Journal of Symbolic Logic 80, 341-358.

[12] Dershowitz, N., Manna, Z., 1979. Proving termination with multiset orderings. Communications of the Association for Computing Machinery $22,465-476$.

[13] Dunn, J.M., Hardegree, G.M., 2001. Algebraic Methods in Philosophical Logic. volume 41 of Oxford Logic Guides. Oxford University Press, Oxford.

[14] Fuchs, L., 1963. Partially Ordered Algebraic Systems. Pergamon Press, Oxford.

[15] Galatos, N., Jipsen, P., Kowalski, T., Ono, H., 2007. Residuated Lattices: An Algebraic Glimpse at Substructural Logics. volume 151 of Studies in Logic and the Foundations of Mathematics. Elsevier, Amsterdam.

[16] Gehrke, M., Harding, J., 2001. Bounded lattice expansions. Journal of Algebra 238, 345-371.

[17] Gehrke, M., Jónsson, B., 1994. Bounded distributive lattices with operators. Mathematica Japonica 40, $207-215$.

[18] Hájek, P., 1998. Metamathematics of Fuzzy Logic. volume 4 of Trends in Logic. Kluwer, Dordrecht.

[19] Hájek, P., 2005. Making fuzzy description logic more general. Fuzzy Sets and Systems 154, 1-15.

[20] Haniková, Z., 2011. Computational complexity of propositional fuzzy logics, in: Cintula, P., Hájek, P., Noguera, C. (Eds.), Handbook of Mathematical Fuzzy Logic - Volume 2. College Publications, London. volume 38 of Studies in Logic, Mathematical Logic and Foundations, pp. 793-851.

[21] Iemhoff, R., 2017. On the existence of alternative Skolemization methods. IfColog Journal of Logics and their Applications 4, $1075-1086$.

[22] McNaughton, R., 1951. A theorem about infinite-valued sentential logic. Journal of Symbolic Logic 16, 1-13.

[23] Metcalfe, G., Olivetti, N., Gabbay, D.M., 2008. Proof Theory for Fuzzy Logics. volume 36 of Applied Logic Series. Springer.

[24] Pigozzi, D.L., 2003. Partially ordered varieties and quasivarieties. http://orion.math.iastate.edu/dpigozzi/ Revised notes of lectures on joint work with Katarzyna Palasinska given at the CAUL, Lisbon in September of 2003, and at the Universidad Catolica, Santiago in November of 2003.

[25] Restall, G., 2000. An Introduction to Substructural Logics. Routledge, New York.

[26] Xu, Y., Ruan, D., Qin, K., Liu, J., 2003. Lattice-Valued Logic: An Alternative Approach to Treat Fuzziness and Incomparability. volume 132 of Studies in Fuzziness and Soft Computing. Springer. 